\title{
Worauf achten Arbeitgeber im Auswahlprozess von Absolventen wirtschaftswissenschaftlicher Studiengänge? Ergebnisse eines faktoriellen Surveys
}

\author{
Christina Neeß
}

Online publiziert: 28. August 2015

(C) Institut für Arbeitsmarkt- und Berufsforschung 2015

\begin{abstract}
Zusammenfassung Stellen Arbeitgeber neue Mitarbeiter ein, so ist der Auswahlprozess häufig geprägt von Unsicherheit, da sich die tatsächliche Produktivität von Arbeitnehmern vorab nur schwer einschätzen lässt. Dies betrifft insbesondere die Rekrutierung von Berufsanfängern. Der Beitrag widmet sich in diesem Sinne der Frage, auf welche Kriterien Entscheidungsträger in Unternehmen bei der Rekrutierung von Hochschulabsolventen achten. Auf Grundlage humankapital- und signaltheoretischer Überlegungen wird die Annahme getroffen, dass individuelle Kompetenzen der Bewerber entscheidend sind. Vor dem Hintergrund veränderter Hochschulstrukturen in Deutschland, die sich besonders deutlich anhand der neuen Studiengänge und -abschlüsse Bachelor und Master zeigen, wird darüber hinaus untersucht, ob zur Verringerung der Unsicherheit auch die Reputation von Universitäten als Signal in den Auswahlprozess einbezogen werden kann. Die Untersuchung der Fragestellung erfolgt mit einem faktoriellen Survey. Die Befunde hieraus zeigen, dass die individuelle Qualifikation des Bewerbers entscheidenden Einfluss auf die Erfolgschancen nimmt: Arbeitgeber beziehen die Abschlussnote in ihre Entscheidung ein, allerdings nicht in einem stärkeren Maße als andere individuelle Kriterien. So erhöht insbesondere Praxiserfahrung die Wahrscheinlichkeit zu einem Vorstellungsgespräch eingeladen zu werden. Auch Auslandserfahrung und ehrenamtliches Engagement verbessern die Erfolgschancen. Der Hochschulreputation kommt in diesem Prozess nahezu keine Bedeutung zu.
\end{abstract}

C. Neeß $(\square)$

Lehrstuhl für Soziologie und Empirische Sozialforschung,

Friedrich-Alexander-Universität Erlangen-Nürnberg,

Findelgasse 7/9,

90402 Nürnberg, Deutschland

E-Mail: soziologie@wiso.uni-erlangen.de
Schlüsselwörter Hochschulabsolventen · Arbeitsmarkteinstieg · Auswahlkriterien · Arbeitgeberbefragung

\section{What do employers look for during the selection process of graduates from economic degrees of study? Results of a factorial survey}

\begin{abstract}
When employers hire a new staff member, the selection process is heavily characterised by uncertainty as the actual productivity of employees can only be estimated in advance. In particular, this affects the recruiting of entrants. In this respect, the article would like to tackle the question of which criteria the decision makers in companies take into consideration when recruiting university graduates. The assumption can be made based on human capital and signal theoretical considerations, that the applicant's individual skills are a deciding factor. Against the background of changing university structures in Germany that are particularly apparent with the new Bachelor and Master study courses and degrees, it will also be investigated whether the reputation of universities as a signal can also be included in the selection process to reduce the level of uncertainty. The investigation of the question took place with a factorial survey. The findings show that the applicant's individual qualifications have a decisive influence over the chances of success: Employers consider the final grades in their decision, although not in such an extent as with other individual criteria. In this way, practical experience in particular raises the likelihood of being invited to a job interview. Experience abroad and voluntary work also improve the chances of success. In this process, the reputation of the university is of almost no importance.
\end{abstract}


Keywords University graduates - Labour market entry · Selection criteria $\cdot$ Employer survey

\section{Einleitung}

Personalentscheider sehen sich im Auswahlprozess neuer Mitarbeiter ex ante unvollständiger Information hinsichtlich der Produktivität von Bewerbern ausgesetzt (Arrow 1973; Garibaldi 2006; Spence 1973). Diese Informationsasymmetrie betrifft ein suchendes Unternehmen vor allem dann, wenn es um die Rekrutierung von Berufsanfängern geht: Mit vergleichsweise wenig Erfahrung ausgestattet zeigt der Berufseinsteiger in seiner Bewerbung geringfügige verlässliche Information anhand derer ein Personalverantwortlicher die Entscheidung über eine Einstellung treffen kann (Altonji und Pierret 2001). Bei der Gruppe der Hochschulabsolventen kommt dieser Mechanismus vor dem Hintergrund des umgreifenden Wandels, den die deutsche Hochschullandschaft derzeitig erlebt, besonders zum Tragen. Mit der Bologna-Reform hat sich die Studienstruktur in Deutschland flächendeckend verändert, indem Diplom und Magister weitestgehend von neu strukturierten Studiengängen mit Bachelor- und Masterabschlüssen abgelöst wurden. Infolgedessen herrscht vor allem bei der Einschätzung der Bachelor-Abschlüsse derzeitig noch eine große Unsicherheit seitens der Unternehmen bzw. deren Personalverantwortlichen (vgl. z. B. Banscherus et al. 2010). Diese Entwicklungen verstärken somit die ohnehin von Unsicherheit geprägte Rekrutierung von Hochschulabsolventen.

In diesem Sinne stellt sich die Frage, auf welche Bewerberkriterien Arbeitgeber achten und ob die Reputation von Universitäten und Hochschulen im Auswahlprozess Hilfestellung bietet. Der Ruf einer Organisation kann sich über verschiedene Kanäle, wie beispielsweise durch die Presse (Fombrun und Shanley 1990) oder durch Informationen aus dem persönlichen Netzwerk, auf das der nachfragende Akteur zurückgreifen kann, verbreiten (Abraham und Meyer 2012; Buskens und Weesie 2000; Uzzi und Lancaster 2004). Im speziellen Kontext der Hochschulreputation nehmen seit einiger Zeit Hochschulrankings eine bedeutsame Rolle ein, da derartige Rangvergleiche Studieninteressierten, Hochschulmanagern und nicht zuletzt Personalentscheidern mehr Informationen zu den Inhalten und der Qualität einzelner Bildungseinrichtungen liefern sollen (Dill und Soo 2005).

Auf Grundlage dieser Überlegungen greift der vorliegende Beitrag das Problem der Informationsasymmetrie im Auswahlprozess von Hochschulabsolventen auf. Hierbei wird untersucht, auf welche Bewerbermerkmale Entscheider in Unternehmen bei der Vorauswahl potenzieller Mitarbeiter achten. Dabei soll auch geklärt werden, in welchem Ausmaß der Name einer Universität von Personalentscheidern als Informationsmerkmal wahrgenommen und in den Auswahlprozess einbezogen wird. Zur Untersuchung der skizzierten Forschungsfragen werden vor dem Hintergrund humankapital- und signaltheoretischer Überlegungen Hypothesen aufgestellt. Ergänzt werden diese durch Annahmen zur Bedeutung von Netzwerken und dem Einfluss räumlicher Nähe von Organisationen. Die empirische Überprüfung basiert auf Daten einer bundesweiten Befragung unter Fachführungskräften in Unternehmen, bei der erfragt wurde, welche Kriterien bei der Einstellung von Hochschulabsolventen wirtschaftswissenschaftlicher Studiengänge wichtig sind. Für die Fragestellung dieses Beitrags erfolgt die Analyse von Daten aus dem in die Studie integrierten faktoriellen Survey. In dem experimentell angelegten Design haben rund 1000 Befragte die Qualifikation fiktiver Bewerber bewertet. Auf dieser Grundlage wird der Einfluss individueller Kompetenzen im Vergleich zur Bedeutung von Hochschulreputation untersucht.

\section{Unsicherheit im Such- und Auswahlprozess von Hochschulabsolventen}

Entscheidungen im Such- und Auswahlprozess neuer Mitarbeiter sind geprägt von Unsicherheit. Fragt ein Arbeitgeber Arbeitskraft nach, so sieht er sich mangelnder Information hinsichtlich der tatsächlichen Produktivität des potenziellen Angestellten ausgesetzt (Arrow 1973; Spence 1973). Gemäß der Humankapitaltheorie investieren rationale Akteure in Bildung, um die eigene Produktivität zu steigern und damit höhere Löhne am Arbeitsmarkt zu erzielen. Auf Grundlage des vorhandenen Humankapitals können Arbeitgeber so im Auswahlprozess eine Einschätzung bezüglich der Leistungsfähigkeit des Bewerbers vornehmen (Becker 1964; Schultz 1961). Allerdings wissen Arbeitnehmer besser um ihre eigenen Fähigkeiten und ihre Produktivität als ein Arbeitgeber (Garibaldi 2006), dementsprechend entsteht eine Informationsasymmetrie (Akerlof 1970). Arbeitgebern steht lediglich die Information über den Investitionsumfang eines Individuums in sein Humankapital zur Verfügung.

Aus Sicht der Signaltheorie dienen die Merkmale von Bewerbern als Signal, die eine Aussage über die Produktivität des potenziellen Mitarbeiters erlauben. Spence (1973) unterteilt dabei Bewerbermerkmale, mithilfe derer ein Arbeitgeber potenzielle Mitarbeiter vorab einschätzen kann, in personenbezogene Attribute - hierbei handelt es sich um personenimmanente Merkmale, die äußerlich erkennbar, aber unveränderlich sind, z. B. Geschlecht oder Hautfarbe - und Signale. $\mathrm{Zu}$ letzteren zählt beispielsweise die Ausbildung, die der rationale Akteur selbst beeinflussen kann. In diesem Sinne beinhalten die Signale eines Bewerbers bewusste Investitionsentscheidungen, die vom Arbeitgeber wiederum als Grundlage zur Bemessung der Entlohnung herangezogen werden. Eine gezielte Investition in ein Sig- 
nal wie beispielsweise Bildung ruft dabei Kosten hervor. Diese werden als so genannte signalling costs bezeichnet, die in Kauf genommen werden, um nach der Ausbildung am Arbeitsmarkt eine adäquate Rendite zu generieren. Die unterschiedlichen Signale, die Individuen mit den von ihnen getätigten Signalkosten aussenden, können dem Arbeitgeber damit eine Differenzierung des Bewerberfeldes ermöglichen. Zu berücksichtigen gilt es dabei allerdings, dass sich die Höhe dieser Signalkosten nicht ausschließlich nach dem persönlichen Einsatz des jeweiligen Akteurs bemisst. Der Investitionsumfang ist stets auch abhängig von der individuellen Veranlagung. Personen mit einer höheren Begabung lernen beispielsweise leichter und müssen daher einen geringeren zeitlichen Aufwand aufbringen als Individuen, denen die Aneignung von Wissen vergleichsweise schwerer fällt. Signale sind nur dann aussagekräftig zur Unterscheidung zwischen Bewerbern mit unterschiedlichen Fähigkeiten, wenn ,die Signalkosten mit der Produktivität negativ korreliert sind“" (Spence 1973).

Bei der Rekrutierung von Hochschulabsolventen bleiben Personalentscheidern in Unternehmen zur Beurteilung ex ante häufig nur die Merkmale des Bildungsabschlusses, (Altonji und Pierret 2001), da Berufseinsteiger über keine bzw. vergleichsweise wenig praktische Erfahrungen verfügen. Hinsichtlich der Einstellung von Hochschulabsolventen bedeutet dies, dass ein Bewerber durch den sogenannten Prozess des Screenings besser eingeschätzt werden kann. Die Signale lassen Rückschlüsse auf unbeobachtbare Eigenschaften zu (Weiss 1995), auf die der Arbeitgeber aufgrund früherer Erfahrungen, die er mit der Einstellung von Hochschulabsolventen gemacht hat, zurückgreifen kann (Arrow 1973).

Die somit ohnehin von Unsicherheit geprägte Einschätzung von Hochschulabsolventen wird derzeitig in Deutschland vor dem Hintergrund veränderter Rahmenbedingungen in der Hochschullandschaft noch zusätzlich verstärkt. Mit der Bologna-Reform wurden Struktur und Aufbau von Studiengängen an Universitäten und (Fach-)Hochschulen stark modifiziert. Dies zeigt sich in erster Linie anhand der Einführung von Bachelor- und Masterstudiengängen. Begreift man Studienabschlüsse als Institutionen, an deren Inhalten sich Marktakteure orientieren, um verlässliche Entscheidungen treffen zu können (Nee 2005), so ruft die Einführung neuer Studienabschlüsse weitere Verunsicherung hervor, da sich die neuen Studienabschlüsse erst etablieren müssen.

\subsection{Hochschulreputation}

Neben der Kenntnis über die individuellen Kompetenzen erhält der Personalentscheider im Auswahlprozess auch die Information, an welcher Bildungseinrichtung ein Bewerber seinen Abschluss erlangt hat. Demzufolge kann die Reputation der jeweiligen Hochschule ebenfalls als Informations- merkmal herangezogen werden, sofern zwischen einzelnen Hochschulinstitutionen Qualitätsunterschiede erkennbar sind.

Begreift man Reputation als Information über einen Akteur, mithilfe derer positive wie negative Eigenschaften jenes Akteures beschrieben werden (Wilson 1985), so kann sie dazu beitragen Informationsasymmetrien am Markt abzubauen (vgl. z. B. Ellickson 1991; Raub und Weesie 1990). Nach Shrum und Wuthnow (1988) nimmt dabei die Bedeutung von Reputation mit steigender Informationsasymmetrie zu. Fombrun (1996) zufolge hilft der gute Ruf, die Glaubwürdigkeit von Anbietern am Markt zu erhöhen, indem Konsumenten stärker darauf vertrauen können, dass sie die versprochene Leistung auch erhalten. Reputation kann daher als immaterielles Kapital bezeichnet werden (vgl. z. B. Rindova et al. 2005), durch das sich Organisationen am Markt entscheidende Wettbewerbsvorteile verschaffen können (Barney 1991; Fombrun und Shanley 1990).

Die Reputationsinformation über einen Akteur ist öffentlich zugänglich, wenn dessen Ruf beispielsweise über die Medien bekannt und damit für jeden sichtbar gemacht wird. In vielen Fällen tragen Organisationen durch entsprechende Kommunikationsstrategien auch selbst entscheidend zum Aufbau von Reputation bei (Fombrun und Riel 1997; Fombrun und Shanley 1990; Schwalbach 2000). Online-Bewertungssysteme wie e-Bay erzeugen ebenfalls Reputation (Bolton et al. 2004; Diekmann und Wyder 2002). Diese teils durch formale Regeln geschaffene Information über einen Akteur lässt sich als institutionalisierte Reputation bezeichnen (Abraham und Meyer 2012; Abraham et al. 2012). Reputationsinformation kann aber auch informell in sozialen Systemen zwischen Akteuren weitergegeben werden (Burt 2005; Granovetter 1985; Sommerfeld et al. 2008; Sommerfeld et al. 2007).

Im Kontext von Hochschulreputation spielen seit einigen Jahren Hochschulrankings eine zentrale Rolle. Diese unternehmen den Versuch, die Leistung von Bildungseinrichtungen vergleichbar zu machen, um so über die Qualität unterschiedlicher Bildungseinrichtungen zu informieren (Dill und Soo 2005). Die Reputation von Hochschulen kann aber neben solch formalisierten Instrumenten auch informell weitergegeben werden, beispielsweise über Absolventen: Da ein Individuum nicht nur über eine persönliche Reputation verfügt, sondern auch die Reputation einer Gruppe widerspiegeln kann, der es angehört (Tirole 1996), können die Alumni einer Universität als Reputationsträger fungieren. Unabhängig davon, ob es sich um einen formellen oder informellen Informationskanal handelt, aus dem eine Information stammt, ist es für die Entfaltung der Reputationswirkung unerlässlich, dass alle beteiligten Akteure über dieselbe Reputationsinformation verfügen und diese glaubwürdig ist (Milgrom und Roberts 1992).

In der Praxis bedeutet dies, dass gute Schulabsolventen durch die gezielte Wahl einer Top-Universität dem späteren 
Arbeitgeber ihr Potenzial signalisieren können (Opitz 2004, S. 17-21), indem sie eine Universität mit einem guten Ruf in dem Wissen wählen, dass auch Arbeitgeber Kenntnis über die positive Reputation jener Hochschuleinrichtung haben. Dieser Argumentation folgend spielt Reputation in einem hierarchischen Bildungsmarkt (Winston 1999), wie er beispielsweise in der US-Hochschullandschaft vorzufinden ist, eine durchaus bedeutsame Rolle: So zeigt Opitz (2004) auf, dass es eine kleine Zahl von Hochschulen gibt, die sich oftmals über längere Zeit - als Spitzenuniversität behaupten können (vgl. auch Hartmann 2006). Dies resultiert daraus, dass sich jene Hochschulen beispielsweise mithilfe einer generösen Forschungsausstattung einen guten Ruf erarbeiten. Dieser impliziert, in welchem Ausmaß in das Humankapital von Studierenden investiert wird. Gelingt es der Universität, dies auch Arbeitgebern zu verdeutlichen, so ist ein sich selbstverstärkender Prozess zu beobachten, bei dem hochreputierte Institutionen ihre Reputation nicht einbüßen, sondern diese vielmehr häufig noch weiter ausbauen.

\subsection{Aktueller Forschungsstand}

Das Problem der Informationsasymmetrie bei der Einschätzung von Humankapital wurde unter anderen von Franck und Opitz $(2001,2004)$ aufgegriffen. Sie zeigen, wie Hochschuleinrichtungen als Signal für die Filterung von High Potentials dienen können (Franck und Opitz 2004; Opitz 2004). In einem weiteren ihrer Beiträge thematisieren sie die Bedeutung von Studiengebühren als Signal für die Qualität der Hochschuleinrichtung (Franck und Opitz 2001).

Der aktuelle Forschungsstand zur Reputation von Hochschulen zeigt sich insbesondere in Bezug auf Hochschulrankings als sehr vielfältig. Studien zu diesem Thema konzentrieren sich verstärkt auf Studieninteressierte als Zielgruppe, wobei für die USA ,mit ihren in der öffentlichen Meinung fest verankerten Rankings" (Hartmann 2006, S. 456) mehrfach empirisch belegt werden konnte, dass Informationen aus derartigen Rangvergleichen oft bei der Hochschulwahl einbezogen werden (Bowman und Bastedo 2009; McDonough et al. 1998). Auch der selbstverstärkende Effekt, der Hochschulen oftmals über einen längeren Zeitraum zu Topplatzierungen verhilft, konnte empirisch gezeigt werden (Bowman und Bastedo 2009). Nach Dill und Soo (2005) nutzen in angelsächsischen Ländern insbesondere statusaffine Studieninteressierte aus Haushalten mit hohem Einkommen und hohem sozialen Status die Hochschulrankings. Im Hinblick auf weltweite Hochschulvergleiche zeigt sich, dass in erster Linie Studieninteressierte, die im Ausland studieren möchten, auf diese blicken. Dies ist vor allem bei asiatischen Schulabgängern festzustellen (Shin und Toutkoushian 2011). ${ }^{1}$

\footnotetext{
${ }^{1}$ Ein umfassender Literaturüberblick zum Thema Hochschulrankings findet sich z. B. bei Bastedo und Bowman (2010). Daneben gibt es
}

Die Bedeutung von Hochschulreputation für den Einstieg in den Arbeitsmarkt und den weiteren Karriereverlauf wurde unter anderem von McGuinness (2003) untersucht. Die Studie zeigt für das Vereinigte Königreich auf, dass die Arbeitsmarktchancen von Berufseinsteigern in erster Linie von Studienfach und Abschlussnote beeinflusst werden, und nicht so sehr von der gewählten Hochschuleinrichtung. Für Deutschland sollte die Hochschulreputation nach Opitz (2004) ebenfalls in geringerem Ausmaß als Filtermechanismus dienen, da hierzulande keine Eliteuniversitäten die Rankings bestimmen; vielmehr bewegt sich die Qualität der Hochschulen auf einem hohen Durchschnittsniveau (Tietzel et al. 1998). Vor dem Hintergrund der 2005 erstmalig von Bund und Ländern ins Leben gerufenen Exzellenzinitiative stellt sich allerdings die Frage, ob sich zukünftig nicht auch die deutsche Hochschullandschaft in Richtung eines zunehmend vom Wettbewerb bestimmten Systems entwickelt (Hartmann 2006).

Zur Reputation von Hochschulen aus Arbeitgebersicht gibt es neben einschlägigen Rangvergleichen, wie sie zum Beispiel das Hochschulranking des Magazins Wirtschaftswoche erstellt (Rettig 2012), nur wenig Evidenz zur Bedeutung des Rufs von Universitäten und Fachhochschulen. Die Zunahme von Rankings und der steigende Wettbewerb im Hochschulbereich werfen aber die Frage auf, ob die Reputation von Hochschulen nicht auch hierzulande an Einfluss gewinnt. Auf wissenschaftlicher Grundlage finden sich im Kontext von Hochschulreputation Studien zur Reputation von Professoren (Brenzikofer 2002), sowie zu Image und Stakeholder-orientierten Ansätzen, in denen auch die Wirkung von Hochschulreputation auf potenzielle Arbeitgeber von Absolventen thematisiert wird (Habicht 2009).

Die von Unternehmen angelegten Strategien bei der Rekrutierung von Hochschulabsolventen wurden in den vergangenen Jahren in verschiedenen Studien empirisch untersucht (für einen Überblick siehe z. B. Burkhardt et al. 2000). Dabei lässt sich ,insgesamt (...) feststellen, dass zwei Gesichtspunkte mehrheitlich genannt werden: fachliche Qualifizierung und Persönlichkeit“" (Teichler 2007, S. 17). Nimmt man allerdings eine spezifischere Differenzierung einzelner Faktoren vor, so zeigen sich heterogene Befunde, die in unterschiedlichen Studien auch verschieden bewertet wurden. Teichler (2007) resümiert in diesem Sinne, dass es kein einzelnes Kriterium gibt, das im Auswahlprozess von Bewerbern entscheidenden Einfluss nimmt. Stattdessen existiert eine große Anzahl an Faktoren,

\footnotetext{
teils spezifische Fragestellungen zum Thema Hochschulreputation: So untersuchten Lowry und Silver (1996) beispielsweise den Zusammenhang zwischen der Reputation einer einzelnen Fakultät und der gesamten Universität. Für das dort gewählte Beispiel der Politikwissenschaften zeigt sich, dass die Reputation der Gesamtuniversität zwar nicht vollständig, aber in weiten Teilen für den Ruf einer Fakultät verantwortlich ist.
} 
denen von einzelnen Entscheidern in Unternehmen auch unterschiedliche Bedeutung zugestanden wird. Aktuellere Untersuchungen zum Thema des Arbeitsmarkteinstiegs von Hochschulabsolventen vor dem Hintergrund der BolognaReform in Deutschland konzentrieren sich nicht so sehr auf Hochschulreputation. Die Ergebnisse einer regionalen Unternehmensbefragung von Lödermann und Scharrer (2010) liefern beispielsweise Erkenntnisse über die von Arbeitgebern gewünschten Kompetenzen und Qualifikationen von Hochschulabsolventen. Wie Alesi et al. (2010) zeigen, stehen in aktuellen Arbeitgeberbefragungen neben den Erwartungen an die Qualifikation der Absolventen insbesondere die Frage nach Kenntnisstand und Akzeptanz der neuen Studienabschlüsse, Einstiegschancen hinsichtlich Gehalt und Position, sowie Karriereentwicklungschancen im Mittelpunkt. Einige dieser Studien widmen sich dabei ausschließlich dem Bachelor-Abschluss (Judt 2006; Sperling 2008).

Der vorliegende Beitrag setzt sich daher zum Ziel, die Fragen nach den von Arbeitgebern angelegten Kriterien und den potenziellen Einfluss von Hochschulreputation im Auswahlprozess vor dem Hintergrund der neuen Studienstrukturen in Deutschland in einen Zusammenhang zu bringen.

\section{Hypothesen}

Personalverantwortliche in Unternehmen sehen sich bei der Auswahl von Bewerbern, die erstmalig dem Arbeitsmarkt zur Verfügung stehen, im Hinblick auf deren zu erwartende Produktivität einer besonders großen Unsicherheit ausgesetzt. Die Bildung, über die ein Bewerber verfügt, wird dabei als eines der vorrangigen Merkmale bei der Auswahl herangezogen (Altonji und Pierret 2001). Stellenausschreibungen benennen die geforderten Kriterien wie Art und Inhalte des Bildungsabschlusses in der Regel deutlich. Damit bewirbt sich eine hinsichtlich des Bildungsniveaus äußerst homogene Gruppe. Deren Mitglieder sind folglich dem gleichen Arbeitsmarktsegment zuzuordnen, da sie über ein formal identisches Bildungszertifikat, zum Beispiel einen Hochschulabschluss, verfügen (Doeringer und Piore 1971). In einem solch homogenen Arbeitsmarktsegment stellt sich daher nicht die Frage nach dem Bildungsgrad an sich, sondern die im Rahmen des Abschlusses erzielten Leistungen, beispielsweise in Form von Noten, sind von zentraler Bedeutung (Haak und Rasner 2009). Humankapital- und signaltheoretischen Ansätzen zufolge impliziert eine gute Abschlussnote, dass der Bewerber Zeit und Aufwand in seine Ausbildung investiert hat.

Kompetenzen, die durch Tätigkeiten und Erfahrungen neben dem eigentlichen Studium gesammelt werden, können ebenfalls Hinweise auf Leistungsbereitschaft und -vermögen des Bewerbers geben. Hierzu zählen beispielsweise
Auslandserfahrung oder ehrenamtliche Tätigkeiten. Darüber hinaus stellt Praxiserfahrung, die in Form von Praktika oder studienbegleitenden Tätigkeiten erworben werden kann (Falk und Reimer 2007), einen essenziellen Indikator bei der Auswahl von Hochschulabsolventen dar. Diese Kompetenzen nach signaltheoretischen Aspekten als Merkmal für Produktivität aufzufassen, erweist sich allerdings als nur bedingt aussagekräftig: Eine solch zusätzliche Tätigkeit lediglich vorzuweisen, lässt noch keinerlei Aussagen über die Produktivität des Bewerbers zu. Der Arbeitgeber erhält aufgrund der bloßen Tatsache, dass ein Praktikum geleistet wurde, noch keine Information über Inhalt und Qualität des Praktikums. Auch über die tatsächliche Einsatzbereitschaft des Absolventen lassen sich mit dieser Information keine Aussagen treffen. Der Personalentscheider sieht aber, ob ein Bewerber sich Zeit für eine solche Aufgabe genommen und damit in sein Humankapital investiert hat, um praktische Erfahrung zu sammeln oder um seine Sozialkompetenz zu erweitern. So zeigen empirische Befunde auch, dass sowohl Praktika (z. B. Sarcletti 2007) als auch studienbezogene Nebentätigkeiten einen positiven Einfluss auf den Arbeitsmarkteintritt für Hochschulabsolventen nehmen (z. B. Franzen und Hecken 2002; Haug und Kropp 2002). Diese Merkmale lassen sich heranziehen, um die Produktivität potenzieller Mitarbeiter einschätzen und vergleichen zu können. Diesen Annahmen folgend wird Hypothese $\mathrm{H}_{1 \mathrm{a}}$ formuliert: Weisen Absolventen wirtschaftswissenschaftlicher Studiengänge individuelle Merkmale wie eine gute Abschlussnote, Praxiserfahrung, Auslandserfahrung und ehrenamtliches Engagement auf, so werden diese von Fachkräften mit Führungsverantwortung eher bei der Personalauswahl berücksichtigt als Absolventen, die diese individuellen Merkmale nicht vorzeigen. $\left(H_{l d}\right)$

Fasst man die Abschlussnote dabei als aggregiertes Ergebnis zahlreicher, vom Absolventen im Laufe des Studiums erbrachten Einzelleistungen auf, so sollten diese eine besonders starke Signalwirkung nach sich ziehen. Schließlich handelt es sich um quantifizierbare Ergebnisse, die Auskunft über die Fachkompetenz des Bewerbers geben. In diesem Sinne ergänzt $H_{l b}$ den Aspekt der individuellen Fähigkeiten: Das Merkmal „Note“ wird von Fachkräften mit Führungsverantwortung stärker bei der Personalauswahl berücksichtigt als andere individuelle Merkmale des Bewerbers wie beispielsweise Praxis- und Auslandserfahrung sowie ehrenamtliches Engagement. $\left(H_{l b}\right)$

Vor dem Hintergrund der skizzierten Veränderungen in der deutschen Bildungslandschaft, wie der deutlich steigenden Zahl von Bildungsanbietern (Hazelkorn 2007) und der Einführung neuer Studiengänge und -abschlüsse im Zuge der Bologna-Reform, zeigt sich eine erhöhte Unsicherheit bezüglich der Wertigkeit der neuen Bildungszertifikate. Damit wird auch die Einschätzung der individuellen Qualifikation der Hochschulabsolventen als Bewerber in einem 
Unternehmen erschwert. Daher sprechen Gründe dafür, dass der Name der vom Bewerber absolvierten Hochschule Hinweise auf die Qualität der Einrichtung liefern kann. Voraussetzung hierfür ist, dass die verschiedenen Hochschuleinrichtungen unterschiedliche Schwierigkeitsniveaus mit sich bringen. Sofern dies der Fall ist, lässt die gewählte Hochschule Rückschlüsse auf die getätigten Humankapitalinvestitionen zu, da sich daraus Unterschiede bezüglich individueller Eigenschaften wie Intelligenz, Einsatzbereitschaft und Ausdauer ableiten lassen.

Die Information über derartige Unterschiede im Qualitätsniveau wird unter anderem über die Veröffentlichung von Hochschulrankings bekannt. Aufgrund der steigenden, medienwirksam publizierten Zahl von Hochschulrankings, ist es für Unternehmen mit verhältnismäßig geringen Kosten verbunden, sich mithilfe von Hochschulrankings über den Ruf einzelner Hochschulen zu informieren. Dieser Argumentation folgend wird angenommen, dass die Reputation einer Institution als zusätzliches Informationsmerkmal genutzt und berücksichtigt wird, wie Hypothese $\mathrm{H}_{2}$ postuliert: Hochschulabsolventen von hochgerankten Universitäten werden eher zum Vorstellungsgespräch eingeladen als Hochschulabsolventen von niedriger gerankten Universitäten. $\left(\mathrm{H}_{2}\right)$

Wird Hochschulreputation im Auswahlprozess neuer Mitarbeiter berücksichtigt, so kann das Wissen über eine Organisation aus verschiedenen Quellen stammen. Hat ein Entscheider selbst ein Studium absolviert, so ist er über die Qualität der eigenen Hochschule besser informiert als über die Studiengänge anderer Hochschuleinrichtungen. In einem solchen Fall liegt die Form sogenannter bilateraler Reputation vor. Der Akteur nutzt das Wissen von Erfahrungen, die er in der Vergangenheit selbst gemacht hat für aktuell zu treffende Entscheidungen (Rooks et al. 2000). Er verfügt damit über Informationen, die Aufschluss über die gute oder schlechte Qualität einer Einrichtung geben können. Im Kontext von Bildungs- und Karrierewegen liegt dabei die Vermutung nahe, dass der Entscheidungsträger demjenigen Bewerber, der an derselben Hochschule studiert hat, generell positiv gegenübersteht, und eigene negative Erfahrungen eine untergeordnete Rolle spielen. Das verbindende Element - die gemeinsame Zugehörigkeit zu der AlumniGruppe einer Universität - sollte sich vor allem positiv auswirken. Die Fachführungskraft in einem Unternehmen wird Absolventen der gleichen Hochschule beziehungsweise des gleichen Studiengangs in besonderem Maße gewogen sein. Eine solche Verhaltensweise lässt sich theoretisch durch das Vorliegen von Homophilie erklären. Akteure präferieren demnach Beziehungen zu Individuen, die ihnen selbst ähnlich sind. Dies kann sich sowohl auf Werte und Einstellungen einer Person beziehen als auch auf soziodemografische Charakteristika. Hierzu zählen auch Bildung und Beruf (McPherson et al. 2001).
Im Hinblick auf das Wissen über Hochschulen sowie auf verbindende Elemente im Sinne von Homophilie ist die eigene Erfahrung allerdings auf eine bzw. wenige Einrichtungen beschränkt. Reputation im engeren Sinne - die sogenannte multilaterale Reputation (Raub und Weesie 1990) - impliziert die Weitergabe von Informationen über Dritte und kann somit mehr Wissen bereitstellen als dies mit eigenen Erfahrungswerten möglich wäre. Verfügt der Entscheidungsträger im Unternehmen aufgrund eigener Erfahrungen oder multilateraler Reputationsinformation über Wissen $\mathrm{zu}$ einer Hochschule, so sollten Bewerber, die an dieser Einrichtung studiert haben, davon profitieren: Entscheidungsträger, die über bilaterale oder multilaterale Reputationsinformation zu einer Hochschule verfügen, bevorzugen Absolventen, die an jenen Hochschulen studiert haben. $\left(H_{3}\right)$

Wenn Studieninteressierte ein Erststudium aufnehmen, spielen im Entscheidungsprozess für einen bestimmten Studiengang unterschiedlichste Faktoren eine Rolle. Dabei sind potenzielle Studienanfänger, die mit ihrem Wechsel von der Schule an eine Hochschule einen neuen Bildungsabschnitt beginnen, in besonderem Maße auf Informationen zu Studienangeboten und Hochschuleinrichtungen angewiesen. Nur so lässt sich eine fundierte Entscheidung treffen. Zwar nutzen die Schulabgänger ihnen zur Verfügung stehende Quellen wie beispielsweise das Internet, Informationen durch die Hochschule (zum Beispiel Informationstage, schriftliche Informationsquellen, Kontakt zu bereits Studierenden), private Kontakte wie Eltern und Freunde, sowie Beratungsangebote der Agentur für Arbeit beziehungsweise durch die Hochschulen selbst. Allerdings werden im Nachhinein nicht alle dieser Informationsquellen als hilfreich beurteilt (Heine et al. 2009). Die empirischen Befunde zeigen vielmehr, dass sich eine „Mehrheit der Schülerinnen und Schüler (...) ein halbes Jahr vor dem Verlassen der Schule jedoch nicht umfassend auf die anstehende Richtungsentscheidung vorbereitet" fühlt (Heine et al. 2007, S. 47). Bei den Motiven zur Studienwahl wird auch deutlich, dass Rankings als Informationsquelle eine untergeordnete Rolle spielen. Letztendlich ist die Reputation der Hochschule für $34 \%$ der Befragten bei der Studienwahl von Bedeutung, der gute Ruf der Lehrenden folgt mit 32\% (Heine et al. 2009). Darüber hinaus zeigen empirische Befunde, dass die Mobilität von Studienanfängern als gering eingeschätzt werden kann. Bei den Gründen für die Hochschulwahl folgt auf das fachliche Interesse vor allem der Wunsch in der Heimatregion zu bleiben (Krawietz und Heine 2007).

Dementsprechend ist davon auszugehen, dass Erstsemester bei der Wahl des Hochschulortes weniger auf den Ruf der Hochschule achten als Master-Studierende. Diese verfügen aufgrund ihres erfolgreich abgeschlossenen Erst-Studiums über deutlich mehr Informationen zum Hochschulsystem. Auch über die individuellen Präferenzen sollten sich Studierende bei ihrer zweiten Studienwahl besser im Klaren sein. 
Die Wahl eines Master-Studienganges erfolgt damit auf Basis eines deutlich größeren Informationsstandes. Insofern ist anzunehmen, dass die Reputation einer Hochschule bei den Motiven für die Wahl eines Studiengangs an Bedeutung gewinnt und eher in die Entscheidung einbezogen wird als bei dem Bachelorstudium. Demzufolge sollte der Name der Hochschule bei Bewerbern mit einem Master-Abschluss von Entscheidungsträgern in Unternehmen eher Berücksichtigung finden als bei Bewerbern mit einem Bachelor-Abschluss: Der Name der Universität, an der ein Bewerber studiert hat, wird im Auswahlprozess neuer Mitarbeiter eher bei Master-Absolventen als bei Bachelor-Absolventen berïcksichtigt. $\left(H_{4}\right)$

Schließlich findet sich in der geografischen Lage der Hochschuleinrichtung ein weiterer Einflussfaktor im Auswahlprozess von Bewerbern. Mit zunehmender regionaler Nähe sollte auch das Wissen über die Qualität einer Hochschuleinrichtung steigen. Der Austausch zwischen Hochschuleinrichtungen und Arbeitsorganisationen zeigt sich beispielsweise in dem Informationstransfer wissenschaftlicher Erkenntnisse oder daran, dass Hochschulen Studierende ausbilden, die nach Beendigung ihres Studiums dem Arbeitsmarkt zur Verfügung stehen (Fritsch 2007).

Auch die Wahrnehmung der Personalentscheider als Privatperson sollte von Bedeutung sein. Einerseits zeigt sich die Berichterstattung über lokale Einrichtungen in den Medien höher, andererseits steigt mit räumlicher Nähe die Wahrscheinlichkeit, Informationen über eine Einrichtung durch das persönliche Netzwerk zu erlangen. So zeigen empirische Befunde aus einer von Landrum et al. (1999) durchgeführten Untersuchung auf, dass die Einwohner eines Bundesstaates in den USA das Image der in der Region gelegenen Universität als positiv bewerten oder eine neutrale Meinung zu der Bildungseinrichtung haben, diese aber nicht schlecht einschätzen. Unter der Annahme, dass die regionale Nähe auch in Deutschland für die Meinungsbildung über eine Hochschule bedeutsam ist, und einen positiven Einfluss auf die Einschätzung der Qualität der jeweiligen Bildungseinrichtung nimmt, wird Hypothese $\mathrm{H}_{5}$ formuliert: Absolventen mit einem Abschluss einer Hochschule in räumlicher Nähe zur Arbeitsorganisation haben größere Chancen beim Arbeitsmarkteinstieg als Bewerber, die an einer räumlich entfernten Bildungseinrichtung studiert haben. $\left(H_{5}\right)$

\section{Methodische Umsetzung}

In einer repräsentativen deutschlandweiten Erhebung wurden Fachführungskräfte in Unternehmen befragt, welche Kriterien sie bei der Einstellung von Hochschulabsolventen wirtschaftswissenschaftlicher Studiengänge anlegen. Dies beinhaltete unter anderem, welche Kompetenzen von Hochschulabsolventen erwartet und wie die neuen Studiengänge und -abschlüsse eingeschätzt werden (Meyer et al. 2013).
Um die Bedeutung individueller Merkmale von Bewerbern zu untersuchen und dem potenziellen Einfluss von Hochschulreputation gegenüber zu stellen, wurde ein faktorieller Survey in die Studie integriert. Hierbei handelt es sich um ein Verfahren, mit dem hypothetisches Verhalten abfragt wird. Anhand fiktiver Situationsbeschreibungen wird die Einschätzung der Befragten untersucht (Beck und Opp 2001; Rossi und Anderson 1982). Diese hypothetischen Szenarien, die Vignetten genannt werden, unterscheiden sich hinsichtlich der systematischen Variation einzelner Merkmale und werden randomisiert auf die Befragten verteilt (Auspurg et al. 2009). Somit lassen sich die kausalen Effekte der in den Situationsbeschreibungen variierten Merkmale auf die Einschätzung der befragten Personen analysieren. Der faktorielle Survey ermöglicht dabei eine Kombination der Antworten aus den Vignetten mit den personenspezifischen Merkmalen der Befragten. Damit lassen sich auch Interaktionen zwischen den Ebenen untersuchen. Diese Methode, die auch als Vignettenanalyse bezeichnet wird, kommt beispielsweise bei Fragestellungen zur Gerechtigkeitsforschung (Liebig und Mau 2005) zum Einsatz, aber auch im Kontext wirtschaftlicher Transaktionen findet sie Anwendung (vgl. z. B. Abraham und Meyer 2012; Rooks et al. 2000). Wie bei anderen experimentellen Verfahren stellt sich auch bei der Vignettenanalyse die Frage nach der externen Validität. Erste Befunde zeigen jedoch, dass die Methode des faktoriellen Surveys bei entsprechendem Framing der Vignetten über die Untersuchung von Einstellungen hinaus auch eingesetzt werden kann, um das hypothetische Verhalten von Akteuren abzubilden (Nisic und Auspurg 2009). Durch die hier vorliegende Befragung von Personen, die sich in ihrer täglichen Praxis mit der abgefragten Fragestellung beschäftigen, sollte die externe Validität zudem gestärkt werden.

Abbildung 1 zeigt den Eingangstext sowie das Beispiel einer Vignette, wie sie den Befragten vorgelegt wurde. Die Ausgangslage besteht aus einer einfach konstruierten Rekrutierungssituation, in der ein Hochschulabsolvent mit wirtschaftswissenschaftlichem Abschluss als neuer Mitarbeiter eingestellt werden soll. Enthalten ist der Hinweis, dass es sich in jeder der folgenden Situationsbeschreibungen um Berufseinsteiger handelt, die für die Stelle fachlich qualifiziert sind. Damit wird die Homogenität bezüglich des formal gleichen Qualifikationsniveaus simuliert. Zudem wurde eine inhaltliche Unterscheidung getroffen, indem der Hälfte der Befragten Situationsbeschreibungen vorgelegt wurden, in denen der Bewerber über einen Bachelor-Abschluss verfügt. Für die anderen Teilnehmer ging es um die Einschätzung von Hochschulabsolventen mit Master-Abschluss.

Die Vignettendimensionen mit ihren jeweiligen Ausprägungen bleiben dabei in beiden Samples identisch. Bei der Gestaltung der Situationsbeschreibungen wurde darauf geachtet, ausschließlich die Merkmale von Bewerbern auf- 
Abb. 1 Ausgangssituation und Beispielvignette für das Sample Bachelor-Abschluss. Zur besseren Verständlichkeit wurden die variierten Vignettenausprägungen in der Erhebung fett hervorgehoben
Ausgangssituation:

Stellen Sie sich vor, Sie sind für die Einstellung eines neuen Mitarbeiters verantwortlich. Intern haben Sie mit Ihren Kollegen vereinbart, einen Berufseinsteiger einzustellen, der gerade sein wirtschaftswissenschaftliches Bachelor-Studium erfolgreich beendet hat. Sie sichten die eingegangenen Unterlagen von Bewerbern, die fachlich für die Stelle qualifiziert sind.

\section{Situation 1:}

Der Bewerber hat sein Bachelor-Studium mit der Note 2,0 absolviert. Er ist ein Absolvent der Universität Würzburg. Der Bewerber verfügt nicht über Auslandserfahrung und engagiert sich ehrenamtlich. Der Kandidat weist einschlägige Praxiserfahrung von insgesamt sechs Monaten auf.

Wie wahrscheinlich ist es, dass Sie den Kandidaten zu einem Vorstellungsgespräch einladen?

sehr unwahrscheinlich

vielleicht

sehr wahrscheinlich zunehmen, die dem Entscheidungsträger in der ersten Stufe des Personalauswahlprozesses auf Grundlage der Bewerbungsunterlagen ohne großen Aufwand zugänglich sind und einer ersten Einschätzung dienen. Damit wird die Situation einer Vorauswahl skizziert, wie sie Unternehmen im Rekrutierungsprozess bei Eingang der Bewerbungsunterlagen vornehmen. Der Hinweis auf die fachliche Eignung des Bewerbers für die Stelle wird gegeben, damit der Befragte seine Abstufung ausschließlich auf Grundlage der genannten Kompetenzen des Bewerbers trifft. Indem die berufliche Qualifikation somit außer Frage steht, können die zu untersuchenden Faktoren systematisch abgefragt werden. Auf weitere Charakteristika, die sich unter dem Begriff Soft Skills zusammenfassen lassen, und zu denen persönliche Eigenschaften wie beispielsweise Führungsstärke, Teamfähigkeit oder ein selbstsicheres Auftreten zu zählen sind, wird bewusst verzichtet. Diese Kompetenzen sind zwar im Rekrutierungsprozess ebenfalls von großer Bedeutung (vgl. z. B. Lödermann und Scharrer 2010), allerdings können sie erst durch differenziertere Instrumente im weiteren Verlauf des Personalauswahlprozesse (zum Beispiel durch Interviews oder Assessment Center) erkannt und eingeschätzt werden (Lorenz und Rohrschneider 2009).

Die Befragten wurden aufgefordert, für jede Situation anzugeben, wie wahrscheinlich sie den jeweiligen Kandidaten zu einem Vorstellungsgespräch einladen würden. Auf einer Likertskala von 1 (,sehr unwahrscheinlich“) bis 7 (,sehr wahrscheinlich“) konnte das Antwortverhalten abgestuft werden. Tabelle 1 zeigt einen Überblick über die in den Vignetten verwendeten Merkmalen und ihre Ausprägungen, die für die beiden Samples „Bachelorabschluss“ und „Masterabschluss“" gelten. Zu Kompetenzen, die einem Bewerber individuell zugeschrieben werden, zählen die Dimension Note mit drei Abstufungen von sehr guter bis befriedigender Leistung, sowie die Merkmale praktische Erfahrung,
Tab. 1 Übersicht der in den Vignetten verwendeten Dimensionen, ihren Ausprägungen und der Art des Merkmals

\begin{tabular}{ll}
\hline Dimension & Ausprsion \\
\hline Note & $1,3-2,0-2,7$ \\
$\begin{array}{l}\text { Praktische } \\
\text { Erfahrung }\end{array}$ & Drei Monate - sechs Monate \\
$\begin{array}{l}\text { Auslandserfah- } \\
\text { rung }\end{array}$ & Keine - Auslandserfahrung \\
$\begin{array}{l}\text { Ehrenamtliches } \\
\text { Engagement }\end{array}$ & Keines - ehrenamtliches Engagement \\
Universität & Erlangen-Nürnberg - Mannheim - European \\
& $\begin{array}{l}\text { Business School Oestrich-Winkel - Würzburg - } \\
\text { HU Berlin }\end{array}$ \\
\hline
\end{tabular}

Auslandserfahrung und ehrenamtliches Engagement. Diese gehen jeweils als dichotome Variablen in das Design ein. Mit der Dimension Universität soll untersucht werden, ob der Ruf der Hochschule, die der Bewerber besucht hat, einen Einfluss auf die Chance der Einladung zum Vorstellungsgespräch nimmt. Da die Unternehmensbefragung im Auftrag des Fachbereichs Wirtschaftswissenschaften der Universität Erlangen-Nürnberg (FAU) durchgeführt wurde, bestand ein besonderes Interesse daran, Aufschluss über die Reputation der eigenen Einrichtung zu erlangen. Somit ging die FAU als Ausprägung der Dimension Universität in das Design ein. $^{2}$ Mit der Universität Mannheim (MH) wurde eine Hochschuleinrichtung aufgenommen, die im Bereich Wirtschaftswissenschaften in den letzten Jahren stets Topplatzierungen erreichte (vgl. z. B. Ott 1999; Rettig 2012). Auch das aktuelle CHE-Hochschulranking ordnet die Universität

\footnotetext{
${ }^{2}$ Die Teilnehmer waren darüber informiert, dass es sich bei der FAU um den Auftraggeber der Studie handelte. Dieser Faktor ist für die spätere Interpretation der Ergebnisse zu berücksichtigen. Aufgrund des hypothetischen Charakters von Vignettenstudien sollten allerdings keine schwerwiegenden Verzerrungen im Antwortverhalten auftreten.
} 
Mannheim bei wirtschaftswissenschaftlichen Bachelor- und Masterstudiengängen in die jeweiligen Spitzengruppen ein (ZEIT Online 2012). Mit der European Business School Oestrich-Winkel (EBS) wird eine Privatuniversität in das Sample aufgenommen, die im Gesamtüberblick aller Universitäten im Bereich Wirtschaftswissenschaften 2012 eine Platzierung unter den besten zehn Hochschuleinrichtungen erreichte und damit als höchst gerankte Privatuniversität in diesem Rangvergleich abschnitt (Rettig 2012). Die Universität Würzburg (WÜ) dient als Ausprägung, mit der die räumliche Nähe zu Arbeitsorganisationen im Vergleich zur FAU - bei den beiden Bildungseinrichtungen handelt es sich um die größten Universitäten Nordbayerns - operationalisiert wird. Die Humboldt-Universität zu Berlin (HU) wird als Kontrollvariable für eine traditionsreiche Universität mit räumlicher Distanz zur FAU in das Design integriert.

Die Untersuchung konzentriert sich bewusst auf Universitäten und nimmt keine Fachhochschulen in das Design auf, da nicht der Unterschied zwischen Hochschultypen abgefragt werden soll. So wird eine mögliche Verzerrung im Antwortverhalten vorab unterbunden. Ein Befragter könnte schließlich generell einen der beiden Hochschultypen bevorzugen. Aus den vorgestellten Dimensionen und ihren jeweiligen Ausprägungen ergeben sich 120 verschiedene Vignetten, die durch Zufallsauswahl auf 20 Decks verteilt wurden. ${ }^{3}$ Diese wurden aufgrund des Samplesplits dupliziert, womit insgesamt 40 Decks vorliegen. Jeder Befragte erhielt sechs Situationsbeschreibungen zur Beurteilung. Die Reihenfolge der einzelnen Vignetten innerhalb eines jeden Decks blieb gleich.

Um die in Hypothese $\mathrm{H}_{5}$ getroffene Annahme testen zu können, werden Variablen der Befragtenebene in die Analyse einbezogen. Das Wissen über die Qualität einer Hochschuleinrichtung, das auf eigenen Erfahrungen des Befragten beruht, wird durch die Angaben der Teilnehmer zu der von ihnen besuchten Hochschule operationalisiert. Die Frage zum Einfluss von Information über Hochschulen durch zusätzliche Informationskanäle kann aufgrund des Studiendesigns nur für die FAU untersucht werden. Für die Analyse wird die Frage nach der Einschätzung des Qualifikationsniveaus von FAU-Absolventen herangezogen. Somit kann die Untersuchung des postulierten Zusammenhangs zwar keinen Anspruch auf Vollständigkeit erheben. Mithilfe dieser Variablen sollten sich aber zumindest erste Hinweise auf den potenziellen Einfluss zusätzlicher Information finden.

Die Erhebung basiert auf einem Switch-Mode-Verfahren, das aus einem kombinierten Einsatz von telefonischer

\footnotetext{
${ }^{3}$ Die zufällige Verteilung von Vignetten auf Decks ist die gängige Vorgehensweise bei der Erstellung von Decks, die wiederum den Befragten zufällig zugewiesen werden (Auspurg et al. 2009). Die Gesamtzahl von Vignetten berechnet sich aus dem sogenannten Kartesischen Produkt $(3 \times 2 \times 2 \times 2 \times 5=120)$.
}

Eingangsbefragung und Onlineerhebung besteht (Höglinger et al. 2010). Befragt wurden (Fach-)Führungskräfte in deutschen Unternehmen, die angaben, potenziell Wirtschaftswissenschaftler zu beschäftigen. Die Mindestgröße des Betriebes wurde auf 50 Beschäftigte festgelegt, womit die Chance erhöht werden sollte, in den kontaktierten Unternehmen potenzielle Arbeitgeber für Wirtschaftswissenschaftler zu finden. Branchen, in denen die Beschäftigung von Wirtschaftswissenschaftlern als sehr gering angenommen wird, wurden in der Vorauswahl ausgeklammert. ${ }^{4}$ Die Gruppe der befragten Personen bestand nicht ausschließlich aus Personalreferenten, sondern aus Fachführungskräften in Abteilungen, die potenziell Wirtschaftswissenschaftler beschäftigen. Diese Entscheidung beruht auf der Annahme, dass der Auswahlprozess neuer Mitarbeiter in vielen Arbeitsorganisationen letztendlich bei den Verantwortlichen der Fachabteilungen liegt bzw. diese über ein erhebliches Mitspracherecht verfügen. Ein weiteres Argument für die gewählte Vorgehensweise gründet darauf, dass es die Verantwortlichen in den Fachabteilungen sind, die über die Einstellung hinaus die neuen Mitarbeiter in ihrer Entwicklung beobachten und deren Leistungsfähigkeit einschätzen können. Demzufolge sollten insbesondere Entscheider in Fachabteilungen über die Kompetenzen und das Leistungsniveau von Hochschulabsolventen informiert sein. In diesem Sinne wurden mit den Abteilungen Einkauf, Marketing, Vertrieb, Finanzwesen und Personalwesen Organisationseinheiten gewählt, in denen Wirtschaftswissenschaftler nach ihrem Studium häufig eine Anstellung finden. Eine weitere Kategorie ist in der Funktion des Geschäftsführers zu finden, um auch kleineren Betrieben eine Einordnung zu ermöglichen, da diese oftmals nicht über spezialisierte Fachabteilungen verfügen. Für die Metropolregion Nürnberg - hierbei handelt es sich um einen Zusammenschluss von 22 Landkreisen und 11 kreisfreien Städten, die sich eine partnerschaftliche Zusammenarbeit zum Ziel gesetzt hat (Metropolregion Nürnberg 2012) - wurde ein gezieltes Oversampling in der Stichprobe vorgenommen. Dieses Vorgehen liegt in einem übergeordneten Ziel der Studie begründet, insbesondere Aufschluss über die Einschätzung die Qualität der FAU Erlangen-Nürnberg zu erlangen.

Die Rekrutierung der zu befragenden Personen im Switch-Mode-Verfahren gestaltete sich folgendermaßen: In einem ersten Schritt wurden Unternehmen telefonisch kontaktiert. Dabei wurde erfragt, welche der für die Befragung relevanten Abteilungen im Betrieb vorhanden sind. Aus diesen wurde ein Bereich zufällig ausgewählt und um die

\footnotetext{
${ }^{4}$ Die Auswahl der Branchen erfolgte auf Grundlage der „Klassifikation der Wirtschaftszweige 2008“" (Statistisches-Bundesamt 2008). Ausgeschlossen wurden z. B. Branchen wie Land- und Forstwirtschaft sowie Erziehung und Unterricht. Auch Unterkategorien wie Praxen von Psychotherapeuten oder Architekturbüros wurden nicht berücksichtigt.
} 
Verbindung zu einer Führungskraft mit Personalverantwortung aus diesem Bereich gebeten. War die richtige Zielperson zu sprechen, so wurde ihr eine kurze, themenbezogene Eingangsfrage gestellt. Anschließend erfasste der Anrufer die E-Mail-Adresse des Befragten. Direkt nach dem Telefonat erhielt dieser die Zugangsdaten zur Teilnahme an der Online-Befragung.

Insgesamt haben 1018 Personen den Online-Fragebogen - den faktoriellen Survey eingeschlossen - vollständig ausgefüllt. Dabei stammen 407 Teilnehmer aus Unternehmen, die in der Metropolregion Nürnberg ansässig sind. Dies entspricht dem angestrebten Oversampling von $40 \%$. Die meisten Befragten stammen aus der Personalabteilung (33\%), der geringste Anteil stammt aus dem Bereich Einkauf $(7,5 \%){ }^{5} 40,8 \%$ der Befragten sind nach eigenen Angaben seit mehr als 10 Jahren in dem Unternehmen tätig, für das sie arbeiten. $43,8 \%$ der Teilnehmer haben selbst ein wirtschaftswissenschaftliches Studium absolviert. Die Rücklaufquote betrug 18,1\%. (Meyer et al. 2013). ${ }^{6}$

\section{Ergebnisse}

Nach einer kurzen Erläuterung der vorliegenden Datenstruktur werden in diesem Abschnitt die Ergebnisse der multivariaten Analysen vorgestellt. Dabei erfolgt die Überprüfung der Hypothesen auf Grundlage verschiedener Modelle. Tabelle 2 gibt zunächst einen Überblick über die in den Analysen verwendeten Variablen. Dargestellt sind die Ausprägungen der Vignettendimensionen sowie die verwendeten Individualvariablen. Die angegebenen Mittelwerte und Standardabweichungen sollen verdeutlichen, dass alle Ausprägungen nahezu gleichverteilt in die Erhebung eingingen.

Für die multivariaten Modelle ist das Design der faktoriellen Surveys zu beachten, bei dem ein Befragter mehrere Urteile abgibt. In der vorliegenden Erhebung erhielt jeder Teilnehmer sechs Vignetten zur Beurteilung vorgelegt. ${ }^{7}$

\footnotetext{
${ }^{5}$ Die weiteren Befragten verteilen sich auf die Bereiche Finanzwesen $(15,7 \%)$, Geschäftsführung (12,3\%), Vertrieb (11,9\%), Marketing $(10,2 \%)$ und Sonstige $(9,5 \%)$. Der hohe Anteil von Teilnehmern aus der Personalabteilung lässt vermuten, dass bei der Nennung des Themas der so genannte „Gatekeeper“ (Höglinger et al. 2010) die Weiterleitung an die Personalabteilung forciert.

${ }^{6}$ Die Rücklaufquote kann im Vergleich zu anderen Arbeitgeberbefragungen im Kontext des Berufseinstiegs von Hochschulabsolventen, die Alesi et al. (2010) in einem Überblick nennen, als leicht überdurchschnittlich bezeichnet werden.

${ }^{7}$ Für die hier präsentierten Ergebnisse der multivariaten Analysen gehen ausschließlich Befragte ein, die ihre Antworten variiert haben. 110 Teilnehmer, die für alle Situationsbeschreibungen die gleiche Antwort gaben, wurden von der Analyse ausgeschlossen, da zu vermuten ist, dass diese Personen kein Interesse an einer Abstufung ihrer Antworten zeigten. Schätzungen mit allen Teilnehmern zeigen keine Unterschiede zu den hier gezeigten Modellen, in denen die Teilnehmer ausgeschlossen wurden.
}

Tab. 2 Übersicht über die in den multivariaten Analysen verwendeten Vignetten- und Befragtenvariablen

\begin{tabular}{|c|c|c|c|c|c|}
\hline & $\begin{array}{l}\text { Anzahl gül- } \\
\text { tiger Fälle }\end{array}$ & Min. & Max. & $\begin{array}{l}\text { Mittel- } \\
\text { wert }\end{array}$ & $\begin{array}{l}\text { Standard- } \\
\text { abweichung }\end{array}$ \\
\hline \multicolumn{6}{|c|}{ Vignettenvariablen } \\
\hline \multicolumn{6}{|l|}{ Abschlussnote } \\
\hline Note 1,3 & 5448 & 0 & 1 & 0.333 & 0.471 \\
\hline Note 2,0 & 5448 & 0 & 1 & 0.328 & 0.469 \\
\hline Note 2,7 & 5448 & 0 & 1 & 0.340 & 0.474 \\
\hline $\begin{array}{l}\text { Auslandserfah- } \\
\text { rung }\end{array}$ & 5448 & 0 & 1 & 0.503 & 0.500 \\
\hline $\begin{array}{l}\text { Ehrenamtliches } \\
\text { Engagement }\end{array}$ & 5448 & 0 & 1 & 0.506 & 0.500 \\
\hline Praxiserfahrung & 5448 & 0 & 1 & 0.498 & 0.500 \\
\hline Universität & 5448 & & & & \\
\hline Mannheim (MH) & 5448 & 0 & 1 & 0.206 & 0.405 \\
\hline FAU Erl.-Nbg. & 5448 & 0 & 1 & 0.200 & 0.400 \\
\hline $\begin{array}{l}\text { EBS } \\
\text { Oestrich-Winkel }\end{array}$ & 5448 & 0 & 1 & 0.189 & 0.391 \\
\hline Würzburg (WÜ) & 5448 & 0 & 1 & 0.203 & 0.402 \\
\hline HU Berlin & 5448 & 0 & 1 & 0.202 & 0.401 \\
\hline \multicolumn{6}{|c|}{ Befragtenvariablen } \\
\hline Alter & 882 & 24 & 75 & 45.580 & 9.506 \\
\hline $\begin{array}{l}\text { Geschlecht }(1= \\
\text { weiblich) }\end{array}$ & 908 & 0 & 1 & 0.340 & 0.474 \\
\hline \multicolumn{6}{|c|}{ Bildungsabschluss } \\
\hline $\begin{array}{l}\text { Kein } \\
\text { HS-Abschluss }\end{array}$ & 908 & 0 & 1 & 0.329 & 0.470 \\
\hline FH- Abschluss & 908 & 0 & 1 & 0.365 & 0.481 \\
\hline Uni-Abschluss & 908 & 0 & 1 & 0.306 & 0.461 \\
\hline $\begin{array}{l}\text { Absolviertes } \\
\text { Studium }(1= \\
\text { WiWi-Studium })\end{array}$ & 908 & 0 & 1 & 0.414 & 0.493 \\
\hline $\begin{array}{l}\text { Beschäfti- } \\
\text { gungsdauer im } \\
\text { aktuellen Betrieb } \\
(1=\text { mehr als } 10 \\
\text { Jahre) }\end{array}$ & 894 & 0 & 1 & 0.421 & 0.494 \\
\hline Studium in $\mathrm{MH}$ & 908 & 0 & 1 & 0.009 & 0.093 \\
\hline $\begin{array}{l}\text { Studium an der } \\
\text { FAU }\end{array}$ & 908 & 0 & 1 & 0.045 & 0.208 \\
\hline Studium in WÜ & 908 & 0 & 1 & 0.014 & 0.119 \\
\hline $\begin{array}{l}\text { Studium an der } \\
\text { HU }\end{array}$ & 908 & 0 & 1 & 0.006 & 0.074 \\
\hline $\begin{array}{l}\text { Information zur } \\
\text { FAU }\end{array}$ & 908 & 0 & 1 & 0.239 & 0.427 \\
\hline $\begin{array}{l}\text { Samplesplit }(1= \\
\text { Master) }\end{array}$ & 908 & 0 & 1 & 0.511 & 0.500 \\
\hline $\begin{array}{l}\text { Metropolregion } \\
\text { (MP) }(1=\text { inner- } \\
\text { halb der MP) }\end{array}$ & 908 & 0 & 1 & 0.402 & 0.490 \\
\hline
\end{tabular}

Diese Antworten können somit nicht unabhängig voneinander betrachtet werden, es liegt eine Clusterung der Daten vor (Hox et al. 1991; Liebig und Mau 2005), die sich beispielsweise mit der Schätzung robuster Standardfehler kontrollieren lässt (Maas und Hox 2004). Da bei der hier diskutierten Fragestellung neben den Niveaueffekten der Vignettendimensionen auch die Variablen der Individual- 
Abb. 2 „Wie wahrscheinlich ist es, dass Sie den Kandidaten zu einem Vorstellungsgespräch einladen?" - Histogramm zur Verteilung der Antworten im faktoriellen Survey

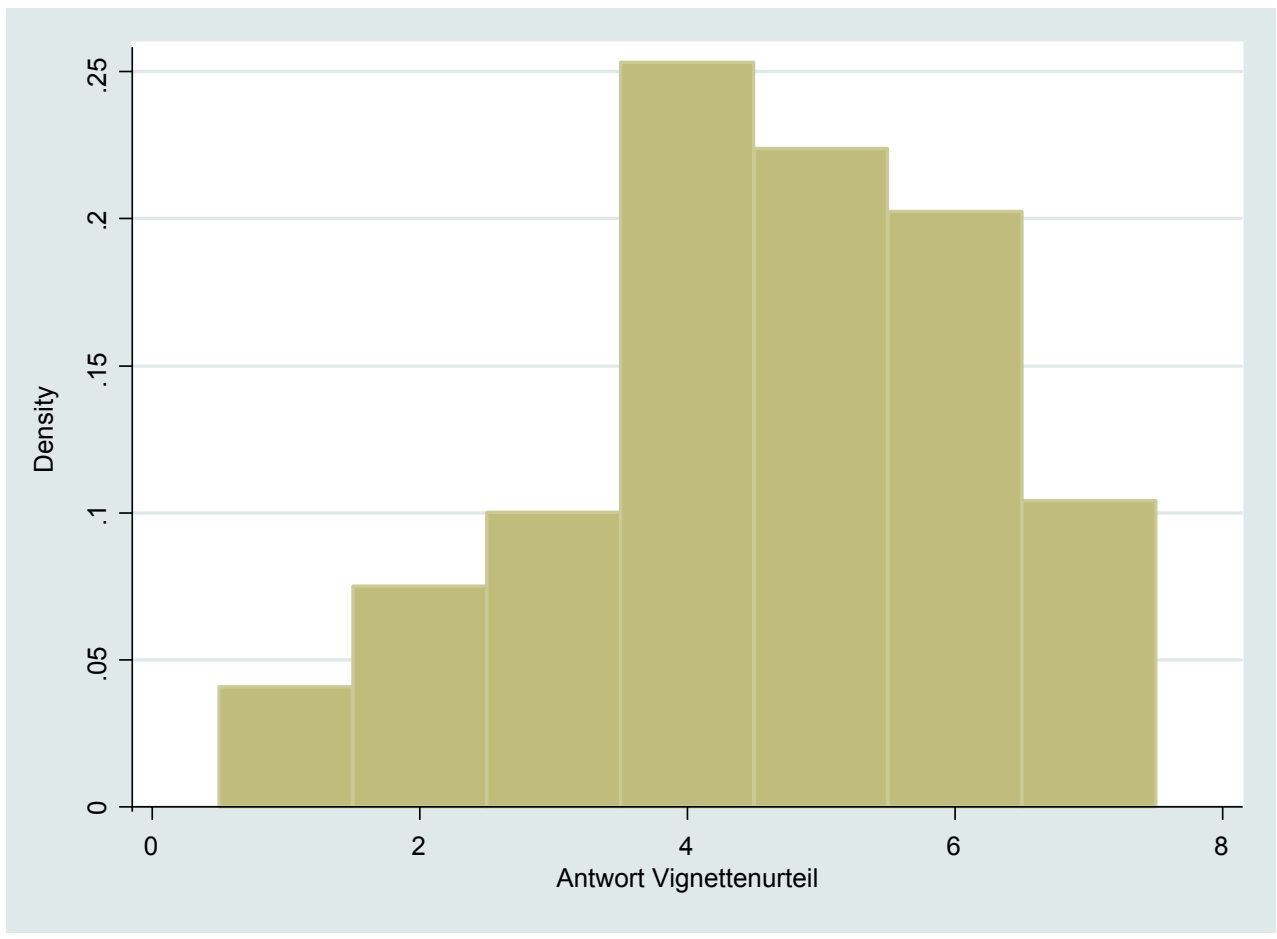

ebene von Interesse sind, wird für die Analysen auf Random-Intercept-Modelle zurückgegriffen (vgl. z. B. Auspurg et al. 2009).

Die Überrepräsentanz der Metropolregion wird durch die Integration eines entsprechenden Gewichtungsfaktors statistisch in der Schätzung der Mixed-Effects-Mehrebenenmodelle berücksichtigt. Mit Ausnahme der Modelle 5 und 6, in denen Befragte außerhalb und innerhalb der Metropolregion getrennt betrachtet werden, ist die Gewichtung in allen Modellgleichungen integriert. In den Modellen wird mit Rho der Anteil der Varianz auf der Befragtenebene an der Gesamtvarianz angegeben. ${ }^{8}$ Die Verteilung der abhängigen Variable wird in Abb. 2 in einem Histogramm dargestellt.

\subsection{Multivariate Ergebnisse des Gesamtmodells}

Die Ergebnisse für das Gesamtsample werden in Tab. 3 im Gesamtmodell 1) dargestellt. Dies dient zur Überprüfung der Hypothesen $\mathrm{H}_{1}, \mathrm{H}_{2}$ und $\mathrm{H}_{3}$. Es zeigt sich, dass die Abschlussnote in die Entscheidung einbezogen wird. Auf Grundlage der in dem Modell verwendeten Variablen werden Bewerber mit einem sehr guten Abschluss auf höchst signifikantem Niveau um 0.198 Punkte auf der Bewertungsskala eher zu einem Vorstellungsgespräch eingeladen als

\footnotetext{
${ }^{8}$ Die abhängige Variable kann nicht als vollständig normalverteilt bezeichnet werden. Dennoch werden die Ergebnisse linearer Schätzungen vorgestellt, da diese die Integration der Gewichtungsfaktoren sowie die Modellierung der Mehrebenenstruktur zulassen. Ordinale Modelle mit der Schätzung robuster Standardfehler kommen in den zentralen Annahmen zu ähnlichen Ergebnissen.
}

Kandidaten, die ein Zeugnis mit der Note ,2,0“ vorweisen können. Der Unterschied zwischen den Noten ,2,0“ und ,2,7“ ist ebenfalls höchst signifikant. Bewerber, die ihr Studium mit der Note ,2,7“ abgeschlossen haben, werden um 0.539 Punkte weniger wahrscheinlich eingeladen als Bewerber mit der Note ,2,0“. Auch die weiteren individuellen Merkmale der Absolventen in den Vignetten nehmen höchst signifikanten Einfluss. Weist ein Kandidat einen Auslandsaufenthalt oder ehrenamtliches Engagement auf, dann steigen seine Chancen auf eine Einladung ebenfalls gegenüber einem Absolventen, der nicht über derartige Erfahrungen verfügt. Bewerber, die Praxiserfahrung vorweisen können, werden ebenfalls eher zu einem Vorstellungsgespräch eingeladen als Bewerber ohne Praxiserfahrung. Hypothese $\mathrm{H}_{1 \mathrm{a}}$ wird damit vorläufig bestätigt.

Zur Überprüfung der Hypothese $\mathrm{H}_{1 \mathrm{~b}}$ bedarf es eines Vergleichs der Effektstärken der einzelnen Variablen, wofür die Koeffizienten paarweise mithilfe von Waldtests verglichen wurden. Erweist sich der getestete Unterschied der beiden Koeffizienten als signifikant, so können Aussagen über die Stärke der Unterschiede zwischen einzelnen Koeffizienten gemacht werden. Die Effektstärken der Dimensionen mit dichotomen Ausprägungen lassen sich jeweils direkt vergleichen. Dabei können die Variablen ehrenamtliches Engagement und Praxiserfahrung auf einem 10\%-Signifikanzniveau als unterschiedlich bezeichnet werden, womit Praxiserfahrung (Koeffizient: 0.543) im Auswahlprozess mehr Bedeutung zukommt als ehrenamtliche Tätigkeit (Koeffizient: 0.438). Die jeweiligen Unterschiede dieser beiden Variablen zur Dimension Auslandserfahrung erwei- 
sen sich nicht als signifikant. Damit können keine statistisch aussagekräftigen Aussagen darüber getroffen werden, ob ein höherer Wert des Koeffizienten auch einen stärkeren Einfluss nimmt. Im jeweiligen Vergleich der Dimension Note mit den beiden Ausprägungen „Note 1,3“ und „Note 2,7“ zur Referenzkategorie „Note 2,0“ zeigen sich die Unterschiede als höchst signifikant. Demnach ist die Ausprägung „Note 1,3“ (Koeffizient: 0.198) im Vergleich zur Note „2,0“ weniger bedeutsam für den Erfolg als das Vorliegen einer Auslandserfahrung (Koeffizient: 0.528) im Vergleich dazu, über keine Auslandserfahrung zu verfügen. Hingegen senkt ein Abschlusszeugnis mit der „Note 2,7“ im Vergleich zu der Merkmalsausprägung „Note 2,0“ (Koeffizient: -0.539) die Chance, zu einem Vorstellungsgespräch eingeladen zu werden in stärkerem Maße als dies der Fall ist, wenn ein Bewerber keine Auslandserfahrung vorweisen kann. Analog lassen sich die Ausprägungen der Dimension Note mit weiteren Koeffizienten vergleichen. Dabei zeigt sich, dass die Koeffizienten der Dimension Note jeweils kleiner sind als die Werte der Dimension Praxiserfahrung. Insbesondere der Unterschied zwischen den Noten „1,3“ und ,2,0“ ist weniger bedeutsam als die anderen integrierten Kompetenzen. Die in Hypothese $\mathrm{H}_{1 \mathrm{~b}}$ aufgestellte Annahme, dass die Note eines Bewerbers im Vergleich zu den Kriterien Auslandserfahrung, ehrenamtliches Engagement und Praxiserfahrung stärker von Entscheidern berücksichtigt wird, bestätigt sich daher nicht. Die Vignettendimensionen Universität nimmt im Gesamtmodell keinen signifikanten Einfluss auf den Auswahlprozess von Hochschulabsolventen. Demnach wird Hypothese $\mathrm{H}_{2}$, die einen Vorteil für Absolventen der Universität Mannheim angenommen hat, abgelehnt. Die vom Bewerber besuchte Hochschule bleibt ohne Effekt im Auswahlprozess.

Für die Universitäten Würzburg und Mannheim zeigt sich, dass Personalentscheider, die an einer dieser beiden Universitäten studiert haben, im Auswahlprozess Bewerber bevorzugen würden, die die gleiche Hochschule besucht haben wie sie. ${ }^{9}$ Dieses Ergebnis deutet darauf hin, dass eine besondere Verbundenheit zur eigenen Alma Mater ausschlaggebend sein kann und in diesen Fällen die theoretisch angenommen Homophilie tatsächlich vorliegt. Verfügt ein Personalentscheider über Informationen aus seinem persönlichen Netzwerk, die ihm Wissen zur Qualifikation von FAU-Absolventen verschaffen, so erhöht sich für Bewerber der FAU die Chance, zum Vorstellungsgespräch eingeladen zu werden, auf schwach signifikantem Niveau. Zieht man dieses Ergebnis zur Überprüfung der in $\mathrm{H}_{3}$ aufgestellten Hypothese heran, so ist zu beachten, dass im Rahmen der

\footnotetext{
${ }^{9}$ Im Fall der EBS Oestrich-Winkel liegen nicht ausreichend Fälle vor, sodass diese Variable nicht in die Analysen eingeht. Für die weiteren Subgruppenanalysen werden die Variablen zur eigenen Erfahrung sowie zur zusätzlichen Information über die FAU nicht in die Modelle integriert, da die Fallzahlen zu gering sind.
}

Erhebung lediglich Informationen zu FAU-Absolventen vorliegen. Die Variable kann somit keinen Anspruch auf Vollständigkeit erheben. Unter Berücksichtigung der mit den Variablen einhergehenden Schwierigkeiten, sowie der Tatsache, dass nicht für alle Universitäten HomophilieEffekte vorliegen, lässt sich Hypothese $\mathrm{H}_{3}$ nicht gänzlich bestätigen und muss daher abgelehnt werden. Die Ergebnisse können lediglich als erster Hinweis darauf verstanden werden, dass eigene Erfahrungen und Informationen durch Dritte einen positiven Einfluss auf die Auswahlwahrscheinlichkeit derjenigen Bewerbern nehmen, über die ein Entscheider Kenntnis besitzt.

Weitere Kontrollvariablen auf der Individualebene bleiben ohne Einfluss auf die Einschätzung der Absolventen durch die Befragten. Lediglich die Variable Wirtschaftswissenschaftliches Studium stellt eine Ausnahme dar: Sind die Befragten selbst Absolventen eines wirtschaftswissenschaftlichen Studienganges, so zeigt sich in allen Modellen, dass diese auf hoch signifikantem Niveau generell eher Bewerber zu einem Vorstellungsgespräch einladen würden als Befragte ohne vergleichbares Studium. Ein möglicher Erklärungsansatz für dieses Ergebnis könnte in der generellen Präferenz der Befragten für Absolventen wirtschaftswissenschaftlicher Studiengänge zu finden sein. Damit läge eine weitere Form von Homophilie vor, die sich nicht auf eine Hochschuleinrichtung, sondern auf eine gemeinsame Fachrichtung bezieht. Dies geht mit Befunden aus der Netzwerkforschung einher, die aufzeigen, dass bei gleichwertiger Bildung und beruflicher Positionen Homophilie vorliegt (für einen Überblick siehe McPherson et al. 2001).

\subsection{Vergleich von Bachelor- und Masterabsolventen}

Betrachtet man das Sample, in dem Situationsbeschreibungen zu Bachelor-Absolventen vorliegen (siehe Tab. 3, Modell 2), so zeigen sich nahezu die gleichen Effekte wie im Gesamtmodell. Bei der Bedeutung von Universitäten ist eine geringfügige Änderung festzustellen, wonach Bachelor-Absolventen der FAU von Befragten tendenziell häufiger zum Vorstellungsgespräch eingeladen werden würden als Bewerber, die einen adäquaten Studienabschluss an der Universität Mannheim erworben haben. Wird das Modell um die Variable zur Information Dritter erweitert, so bleibt dieser Unterschied allerdings nicht mehr bestehen. ${ }^{10}$ In den Urteilen zu Master-Absolventen, die in Modell 3 dargestellt sind, finden sich allerdings Unterschiede zu den vorangegangenen Modellen. Die bei Bachelor-Absolventen vorge-

\footnotetext{
${ }^{10}$ Wie zuvor erläutert liegen keine empirischen Befunde zum Informationsstand der Befragten über die anderen in den Vignetten genannten Universitäten vor. Somit kann nicht untersucht werden, ob ähnliches Wissen nicht auch einen Effekt auf das gesamte Auswahlverhalten nehmen würde. Auf eine Darstellung dieser Ergebnisse sowie auf eine weitere Interpretation zu dieser Variablen wird daher verzichtet.
} 
Tab. 3 Ergebnisse der Mehrebeneschätzungen Modelle 1-4

\begin{tabular}{|c|c|c|c|c|}
\hline Vignetten-Dimensionen & $\begin{array}{l}\text { Gesamtmodell } \\
\text { (1) }\end{array}$ & $\begin{array}{l}\text { Sample Bachelor-Abschluss } \\
\text { (2) }\end{array}$ & $\begin{array}{l}\text { Sample Master-Abschluss } \\
\text { (3) }\end{array}$ & $\begin{array}{l}\text { Vergleich Bachelor-/Master-Ab- } \\
\text { schluss (4) }\end{array}$ \\
\hline \multicolumn{5}{|l|}{$\overline{\text { Note (Ref.: 2,0) }}$} \\
\hline \multirow[t]{2}{*}{1,3} & $0.198 * * *$ & $0.316 * * *$ & 0.083 & $-0.233^{* *}$ \\
\hline & $(0.043)$ & $(0.065)$ & $(0.057)$ & $(0.086)$ \\
\hline \multirow[t]{2}{*}{2,7} & $-0.539 * * *$ & $-0.536^{* * *}$ & $-0.546^{* * *}$ & -0.010 \\
\hline & $(0.050)$ & $(0.071)$ & $(0.069)$ & $(0.010)$ \\
\hline Auslandserfahrung & $0.528 * * *$ & $0.517 * * *$ & $0.538 * * *$ & 0.020 \\
\hline (Ref.: keine) & $(0.045)$ & $(0.066)$ & $(0.060)$ & $(0.090)$ \\
\hline Ehrenamt & $0.438 * * *$ & $0.394 * * *$ & $0.479 * * *$ & 0.083 \\
\hline (Ref.: Keines) & $(0.045)$ & $(0.064)$ & $(0.063)$ & $(0.090)$ \\
\hline Praxiserfahrung & $0.543^{* * *}$ & $0.554 * * *$ & $0.536^{* * *}$ & -0.020 \\
\hline (Ref.: 2 Monate) & $(0.046)$ & $(0.069)$ & $(0.063)$ & $(0.093)$ \\
\hline \multicolumn{5}{|l|}{ Universität } \\
\hline \multicolumn{5}{|l|}{ (Ref.: Mannheim) } \\
\hline \multirow[t]{2}{*}{ FAU } & 0.028 & $0.193 *$ & -0.102 & $-0.295^{*}$ \\
\hline & $(0.064)$ & $(0.086)$ & $(0.079)$ & $(0.117)$ \\
\hline \multirow[t]{2}{*}{ EBS } & -0.103 & 0.009 & $-0.231^{*}$ & -0.242 \\
\hline & $(0.068)$ & $(0.101)$ & $(0.091)$ & $(0.136)$ \\
\hline \multirow[t]{2}{*}{ Würzburg } & -0.086 & 0.005 & $-0.189^{*}$ & -0.193 \\
\hline & $(0.058)$ & $(0.083)$ & $(0.080)$ & $(0.115)$ \\
\hline \multirow[t]{2}{*}{ HU Berlin } & -0.083 & 0.006 & $-0.190^{*}$ & -0.197 \\
\hline & $(0.058)$ & $(0.085)$ & $(0.079)$ & $(0.116)$ \\
\hline \multicolumn{5}{|l|}{ Individualebene } \\
\hline Geschlecht & 0.049 & -0.134 & 0.235 & 0.369 \\
\hline (Ref.: Männlich) & $(0.097)$ & $(0.132)$ & $(0.141)$ & $(0.192)$ \\
\hline Studium WiWi & $0.305^{* *}$ & $0.364 *$ & 0.261 & -0.103 \\
\hline (Ref.: keines) & $(0.112)$ & $(0.154)$ & $(0.158)$ & $(0.221)$ \\
\hline Metropolregion & -0.084 & 0.070 & -0.103 & -0.157 \\
\hline (Ref.: außerhalb) & $(0.090)$ & $(0.107)$ & $(0.114)$ & $(0.205)$ \\
\hline Samplesplit & 0.013 & & & \\
\hline (Ref.: $B A)$ & $(0.090)$ & & & \\
\hline Information & 0.051 & & & \\
\hline über FAU & $(0.126)$ & & & \\
\hline Information über FAU* & $0.225^{*}$ & & & \\
\hline Vig. $F A U$ & $(0.114)$ & & & \\
\hline Studienort Mannheim & 0.098 & & & \\
\hline$(\mathrm{MH})$ & $(0.263)$ & & & \\
\hline Studienort & 0.530 & & & \\
\hline FAU & $(0.271)$ & & & \\
\hline Studienort & 0.106 & & & \\
\hline Würzburg (WÜ) & $(0.351)$ & & & \\
\hline Studium $\mathrm{MH}^{*}$ & $1.044^{*}$ & & & \\
\hline Vig. $M H$ & $(0.486)$ & & & \\
\hline Studium FAU* & -0.270 & & & \\
\hline Vig. $F A U$ & $(0.238)$ & & & \\
\hline Studium WÜ* & $0.588 * * *$ & & & \\
\hline Vig. $W \ddot{U}$ & $(0.153)$ & & & \\
\hline \multirow[t]{2}{*}{ Konstante } & $4.268 * * *$ & $4.814 * * *$ & $3.945 * *$ & \\
\hline & $(0.827)$ & $(1.093)$ & $(1.213)$ & \\
\hline$N$ & 5232 & 2598 & 2634 & \\
\hline
\end{tabular}


Tab. 3 (Fortsetzung)

\begin{tabular}{|c|c|c|c|c|}
\hline Vignetten-Dimensionen & $\begin{array}{l}\text { Gesamtmodell } \\
\text { (1) }\end{array}$ & $\begin{array}{l}\text { Sample Bachelor-Abschluss } \\
\text { (2) }\end{array}$ & $\begin{array}{l}\text { Sample Master-Abschluss } \\
\text { (3) }\end{array}$ & $\begin{array}{l}\text { Vergleich Bachelor-/Master-Ab- } \\
\text { schluss (4) }\end{array}$ \\
\hline \multicolumn{5}{|l|}{ Random-Effect Parameters } \\
\hline \multirow[t]{2}{*}{$\operatorname{sd}\left(\_\right.$cons $)$} & 0.985 & 0.938 & 0.026 & \\
\hline & $(0.036)$ & $(0.053)$ & $(0.050)$ & \\
\hline \multirow[t]{2}{*}{ sd(Residual) } & 1.016 & 1.027 & 1.005 & \\
\hline & $(0.021)$ & $(0.030)$ & $(0.030)$ & \\
\hline Rho & 0.485 & 0.455 & 0.511 & \\
\hline
\end{tabular}

Random-Intercept-Modell mit Gewichtung für die Variable Metropolregion. Abhängige Variable „Wie wahrscheinlich würden Sie den Bewerber zum Vorstellungsgespräch einladen?“ (Likertskala von 1 „Sehr unwahrscheinlich“ bis 7 „Sehr wahrscheinlich“)

Signifikant für: $p<0.001(* * *) ; p<0.01(* *) ; p<0.05(*)$. Robuste Standardfehler in Klammern. In den Modellen wird zusätzlich für die Individualvariablen Alter, Alter ${ }^{2}$, Bildungsstand, Beschäftigungsdauer, Eigener Hochschulort, HU Berlin sowie dessen Interaktionseffekt kontrolliert, hierbei zeigen sich keine signifikanten Ergebnisse

nommene Differenzierung von Bewerbern im Hinblick auf ihre Abschlussnote bleibt bei der Einschätzung von Master-Absolventen nur noch teilweise bestehen: Es zeigen sich keine signifikanten Unterschiede zwischen Bewerbern, die mit der Note „1,3“ ihr Studium abgeschlossen haben, und denjenigen, die eine „2,0“ im Zeugnis vorweisen können. Beide werden in gleichem Maße zu Vorstellungsgesprächen eingeladen. Ein statistischer Vergleich der beiden Samples Bachelor-Abschluss und Master-Abschluss (siehe Modell 4) zeigt, dass die Unterschiede im Verhalten zwischen den beiden Samples hinsichtlich der Bedeutung der Note als signifikant zu bezeichnen sind.

Bei der Auswahl von Bewerbern im Sample Master-Abschluss wird der Name der Universität eher herangezogen als im Sample Bachelor-Abschluss. Die Befragten unterscheiden nicht zwischen Hochschulabsolventen der Universitäten Mannheim und Erlangen-Nürnberg, sie würden aber signifikant weniger wahrscheinlich Bewerber der anderen drei Hochschulen zum Vorstellungsgespräch bitten. Bei der Auswahl von Master-Absolventen scheint der Name der Hochschule somit tendenziell eine Rolle zu spielen.

Im Vergleich der beiden Samples (Modell 4) ${ }^{11}$ zeigt sich, dass die Unterschiede zwischen den Samples Bachelor- und Master-Abschluss mit Ausnahme der FAU nicht als signifikant eingestuft werden können. Hypothese $\mathrm{H}_{4}$, wonach Entscheidungsträger im Unternehmen eher bei Master- als bei Bachelor-Absolventen den Namen der Hochschule berücksichtigen, bestätigt sich demnach nicht.

\subsection{Vergleich innerhalb und außerhalb der Metropolregion}

Die in Hypothese $\mathrm{H}_{5}$ getroffene Annahme der Bedeutung räumlicher Nähe wird mit einem weiteren Vergleich der Samples außerhalb der Metropolregion (Modell 5) und

\footnotetext{
${ }^{11}$ Der Vergleich wurde mit der Integration von Interaktionseffekten zwischen den beiden Modellen umgesetzt. Eine analoge Vorgehensweise findet sich in Modell 7.
}

Metropolregion (Modell 6) untersucht. Die Ergebnisse werden in Tab. 4 präsentiert. Im Mittelpunkt der Analysen steht die Frage, ob Befragte aus der Metropolregion Absolventen der Universitäten Erlangen-Nürnberg und Würzburg, also Hochschuleinrichtungen in räumlicher Nähe, bevorzugen.

Zunächst zeigt sich, dass Befragte außerhalb der Metropolregion signifikant mehr Wert auf Auslandserfahrung legen als Mitarbeiter von Unternehmen innerhalb der Metropolregion. In beiden Samples sind die ausschlaggebenden Entscheidungskriterien wiederum in den individuellen Merkmalen der Bewerber zu finden, während der Name der Hochschule keine Rolle spielt. Im relativen Vergleich der einzelnen Universitäten zeigen sich jedoch Unterschiede: Während im überregionalen Sample kein signifikanter Unterschied zwischen der FAU und der Universität Mannheim zu finden ist, erfährt die FAU innerhalb der Metropolregion eine größere Zustimmung; es würden signifikant häufiger Bewerber der FAU eingeladen werden als Kandidaten mit einem Abschluss der Universität Mannheim. Der Unterschied zwischen den beiden Gruppen außerhalb und innerhalb der Metropolregion ist allerdings nicht signifikant.

Für die Universität Würzburg zeigen sich keine signifikanten Effekte, allerdings verändert sich das Vorzeichen des Koeffizienten von einem negativen Wert im Sample außerhalb Metropolregion zu einem positiven im Sample Metropolregion. Dieser Unterschied ist im Modell zum Vergleich der beiden Samples (Modell 7) als schwach signifikant zu bezeichnen. Zwar deuten die Befunde darauf hin, dass die regionale Nähe einen positiven Effekt auf die Auswahl von Bewerbern nimmt. Die Hypothese wird aufgrund der vorliegenden empirischen Befunde aber nur in Teilen bestätigt. Daher wird auch Hypothese $\mathrm{H}_{5}$ abgelehnt.

\section{Fazit}

Das Ziel des vorliegenden Beitrags bestand darin, Aufschluss über die Kriterien zu erlangen, die Entschei- 
Tab. 4 Ergebnisse der Mehrebenenschätzungen Modelle 5-7

\begin{tabular}{|c|c|c|c|}
\hline $\begin{array}{l}\text { Vignetten-Di- } \\
\text { mensionen }\end{array}$ & $\begin{array}{l}\text { e Metro- } \\
\text { gion (5) }\end{array}$ & $\begin{array}{l}\text { Metropolre- } \\
\text { gion (6) }\end{array}$ & $\begin{array}{l}\text { Vergleich zw. } \\
\text { Keine Metropol- } \\
\text { region - Metro- } \\
\text { polregion ( } 7)\end{array}$ \\
\hline \multicolumn{4}{|l|}{ Note $($ Ref.: 2,0$)$} \\
\hline 1,3 & $\begin{array}{l}0.199 * * * \\
(0.046)\end{array}$ & $\begin{array}{l}0.216^{* * *} \\
(0.060)\end{array}$ & $\begin{array}{c}0.175 \\
(0.075)\end{array}$ \\
\hline 2,7 & $\begin{array}{l}-0.545^{* * *} \\
(0.047)\end{array}$ & $\begin{array}{l}-0.450 * * * \\
(0.061)\end{array}$ & $\begin{array}{c}0.095 \\
(0.752)\end{array}$ \\
\hline $\begin{array}{l}\text { Auslandserfahrung } \\
\text { (Ref.: keine) }\end{array}$ & $\begin{array}{l}0.533 * * * \\
(0.040)\end{array}$ & $\begin{array}{l}0.377 * * * \\
(0.051)\end{array}$ & $\begin{array}{c}-0.156^{*} \\
(0.076)\end{array}$ \\
\hline $\begin{array}{l}\text { Ehrenamt } \\
\text { (Ref.: Keines) }\end{array}$ & $\begin{array}{l}0.433 * * * \\
(0.039)\end{array}$ & $\begin{array}{l}0.523 * * * \\
(0.051)\end{array}$ & $\begin{array}{c}0.090 \\
(0.077)\end{array}$ \\
\hline \multicolumn{4}{|l|}{$\begin{array}{l}\text { Universität } \\
\text { (Ref.: Mannheim) }\end{array}$} \\
\hline FAU & $\begin{array}{c}0.038 \\
(0.063)\end{array}$ & $\begin{array}{c}0.196^{*} \\
(0.081)\end{array}$ & $\begin{array}{c}0.159 \\
(0.103)\end{array}$ \\
\hline EBS & $\begin{array}{c}-0.106 \\
(0.062)\end{array}$ & $\begin{array}{l}-0.311^{* * * *} \\
(0.079)\end{array}$ & $\begin{array}{c}-0.206 \\
(0.114)\end{array}$ \\
\hline Würzburg & $\begin{array}{c}-0.105 \\
(0.061)\end{array}$ & $\begin{array}{c}0.135 \\
(0.078)\end{array}$ & $\begin{array}{c}0.240^{*} \\
(0.102)\end{array}$ \\
\hline HU Berlin & $\begin{array}{c}-0.093 \\
(0.062)\end{array}$ & $\begin{array}{c}-0.108 \\
(0.080)\end{array}$ & $\begin{array}{c}-0.014 \\
(0.099)\end{array}$ \\
\hline \multicolumn{4}{|l|}{ Individualebene } \\
\hline $\begin{array}{l}\text { Geschlecht } \\
\text { (Ref.: Männlich) }\end{array}$ & $\begin{array}{c}0.041 \\
(0.101)\end{array}$ & $\begin{array}{c}0.118 \\
(0.137)\end{array}$ & $\begin{array}{c}0.078 \\
(0.165)\end{array}$ \\
\hline $\begin{array}{l}\text { Studium WiWi } \\
\text { (Ref.: keines) }\end{array}$ & $\begin{array}{l}0.319 * * \\
(0.116)\end{array}$ & $\begin{array}{c}0.069 \\
(0.163)\end{array}$ & $\begin{array}{c}-0.251 \\
(0.196)\end{array}$ \\
\hline $\begin{array}{l}\text { Samplesplit } \\
\text { (Ref.: } B A \text { ) }\end{array}$ & $\begin{array}{c}0.017 \\
(0.095)\end{array}$ & $\begin{array}{c}-0.160 \\
(0.121)\end{array}$ & $\begin{array}{c}-0.160 \\
(0.121)\end{array}$ \\
\hline Konstante & $\begin{array}{l}4.317 * * * \\
(0.957)\end{array}$ & $\begin{array}{l}4.565^{* * * *} \\
(1.159)\end{array}$ & \\
\hline$N$ & 3102 & 2130 & 5232 \\
\hline \multicolumn{4}{|c|}{ Random-Effect Parameters } \\
\hline sd(_cons) & $\begin{array}{l}0.988 \\
(0.036)\end{array}$ & $\begin{array}{l}1.042 \\
(0.046)\end{array}$ & \\
\hline sd(Residual) & $\begin{array}{l}1.015 \\
(0.014)\end{array}$ & $\begin{array}{l}1.084 \\
(0.018)\end{array}$ & \\
\hline Rho & 0.486 & 0.480 & \\
\hline
\end{tabular}

Random-Intercept-Modell mit Gewichtung für die Variable Metropolregion für den Gruppenvergleich

Abhängige Variable „Wie wahrscheinlich würden Sie den Bewerber zum Vorstellungsgespräch einladen?“ (Likertskala von 1 „Sehr unwahrscheinlich“ bis 7 ,Sehr wahrscheinlich“.)

Signifikant für: $\left.p<0.001(* * *) ; p<0.01{ }^{* *}\right) ; p<0.05\left(^{*}\right) ; p<0.1$ $(+)$. Robuste Standardfehler in Klammern. In den Modellen wird zusätzlich für die Individualvariablen Alter, Alter ${ }^{2}$, Bildungsstand sowie Beschäftigungsdauer, kontrolliert, für diese zeigen sich keine signifikanten Effekte

dungsträger in deutschen Unternehmen derzeitig bei der Auswahl von Bewerbern mit wirtschaftswissenschaftlichem Abschluss anlegen. Dies zum jetzigen Zeitpunkt zu untersuchen, scheint sinnvoll: Die Bologna-Reform hat die deut- schen Hochschulstrukturen nachhaltig verändert und mit der Einführung von Bachelor- und Masterstudiengängen drängen nun seit kurzem Absolventen mit neuen Abschlüssen auf den Arbeitsmarkt. Dementsprechend lässt sich mithilfe der Untersuchung aufzeigen, welche Bewerbermerkmale Arbeitgeber in der aktuellen Situation wahrnehmen und worauf sie besonderen Wert legen. Neben den individuellen Merkmalen, die Hochschulabsolventen mitbringen, wurde auch die Frage untersucht, ob die Reputation einer Universität Einfluss auf den Auswahlprozess nimmt.

Die Ergebnisse zeigen, dass Bewerber weiterhin auf Grundlage ihrer individuellen Qualifikation eingeschätzt werden. Mit einem guten Studienabschluss, mit Praxis- und Auslandserfahrung sowie ehrenamtlichem Engagement stehen die Chancen für Absolventen gut, zu einem Vorstellungsgespräch eingeladen zu werden. Der Name der Hochschule spielt hingegen zum jetzigen Zeitpunkt für deutsche Fachführungskräfte eine eher untergeordnete Rolle bei der Personalauswahl. Entscheidungsträger in Unternehmen scheinen allerdings bei der Auswahl von Master-Studierenden den Namen der Hochschule, an der ein Bewerber studiert hat, eher in ihre Entscheidung einzubeziehen: Die Ergebnisse für die Universität Mannheim im faktoriellen Survey zeigen, dass deren Alumni im Gegensatz zu Absolventen anderer Hochschulen leicht bevorzugt werden. Demnach scheinen die Ergebnisse von Hochschulrankings, nach denen die Universität Mannheim seit langem als Spitzenuniversität im Bereich Wirtschaftswissenschaften gilt, von Personalverantwortlichen nicht gänzlich ignoriert zu werden. Verhältnisse wie im US-Hochschul- und Arbeitsmarkt, in dem Top-Universitäten bereits über ihren Namen verdeutlichen, für welche Positionen am Arbeitsmarkt ihre Absolventen in Frage kommen, sind in Deutschland auf Grundlage der vorliegenden Ergebnissen derzeitig aber nicht zu finden. Für Entscheider hierzulande stehen weiterhin die individuellen Merkmale von Bewerbern an erster Stelle. Die Annahme, dass der Abschlussnote dabei eine größere Bedeutung zukommt als anderen individuellen Kompetenzen, bestätigt sich nicht. Es zeigt sich aber, dass es für Entscheider bei der Auswahl von Master-Absolventen nicht von Bedeutung ist, ob ein sehr guter Abschluss erlangt wurde; lediglich Bewerber mit einer Abschlussnote schlechter als 2,0 erfahren im Auswahlprozess Nachteile.

Die Ergebnisse des vorliegenden Beitrags verdeutlichen außerdem, dass der individuelle Kenntnis- und Informationsstand des Entscheiders im Unternehmen Einfluss auf die Auswahlwahrscheinlichkeit nimmt. Der theoretisch angenommenen Bedeutung von persönlich generiertem Wissen - sowohl durch eigene Erfahrung als auch durch die Informationen über Dritte - zu einer Hochschule kann zwar mit den vorliegenden Daten nicht vollständig überprüft werden. Dennoch erlauben die Ergebnisse den Eindruck, dass zusätzliches Wissen Einfluss auf den Rekrutierungsprozess 
nehmen kann, beziehungsweise Homophilie-Effekte vorliegen. Der Unternehmensstandort scheint keine bedeutsame Rolle im Auswahlprozess zu spielen. Absolventen regionaler Universitäten genießen gegenüber Mitbewerbern, die an entfernter gelegenen Bildungseinrichtungen studiert haben, im Auswahlprozess bestenfalls leichte Vorteile.

Aufgrund des Designs, auf dem die vorliegenden Daten beruhen, ist es nicht auszuschließen, dass Reputationseffekte und damit auch die Bedeutung von Hochschulrankings in Deutschland, unterschätzt werden: Es handelt sich um ein faktorielles Design, das Verhalten stets hypothetisch abfragt, und dem eine Auswahl von Hochschulen vorangegangen ist. Mit der Festlegung auf eine begrenzte Anzahl variierter Dimensionen vermag es diese Methode auch nicht, den gesamten Such-, Auswahl- und Einstellungsprozess von Bewerbern vollständig abzubilden. Lediglich ein Ausschnitt des gesamten Recruitingprozesses konnte in der vorliegenden Studie simuliert werden.

In diesem Kontext stellt sich allerdings auch die Frage, inwiefern Hochschulrankings, die in großem Maße für die Information über die Reputation einer Hochschule verantwortlich sind, echte Vergleiche vornehmen und aufzeigen können. Methodische und inhaltliche Probleme bei der Erstellung dieser Rangvergleiche sind evident, sodass Zweifel an der Validität dieser Instrumente bestehen bleiben. Dies zeigt sich beispielsweise in der im Juni 2012 abgegebenen Empfehlung der DGS an Soziologie-Studiengänge, sich nicht mehr an einer Evaluierung durch das CHE-Ranking zu beteiligen (DGS 2012). Auch die in der vorliegenden Arbeitgeberbefragung an anderer Stelle direkt gestellte Frage, inwiefern die Befragten Rankings als hilfreich einschätzen, wenn es um die Rekrutierung von Hochschulabsolventen geht, zeigt, dass knapp ein Drittel der befragten Fachführungskräfte Rankings als wenig hilfreich einschätzen. 14,8\% gaben an, den Nutzen nicht beurteilen zu können, (Meyer et al. 2013), womit deutlich wird, dass Rankings aktuell lediglich in geringem Umfang wahrgenommen und genutzt werden. Diese Faktoren könnten erklären, warum in den vorgelegten Vignetten der Name der Hochschule wenig Einfluss auf die Entscheidung nimmt. Im Hinblick auf einen zunehmenden Wettbewerb in der nationalen wie internationalen Bildungslandschaft bleibt zu beobachten, ob sich der Stellenwert von Hochschulreputation in den kommenden Jahren in Deutschland ändern wird und inwiefern Arbeitgeber im Auswahlprozess von Hochschulabsolventen darauf reagieren werden.

\section{Kurzfassung}

Bei der Auswahl und Einstellung neuer Mitarbeiter sehen sich Personalentscheider häufig großer Unsicherheit ausgesetzt, da sich die Einschätzung der tatsächlichen Produk- tivität eines Arbeitnehmers vorab oft schwierig gestaltet. Die so ex ante bestehende Informationsasymmetrie zum Nachteil des Arbeitgebers wird vor allem bei der Rekrutierung von Berufsanfängern deutlich, denn diese weisen eine vergleichsweise geringfügige verlässliche Information auf, anhand derer ein Personalverantwortlicher die Entscheidung über eine Einstellung treffen kann. Diese Problematik kommt hierzulande besonders bei der Gruppe der Hochschulabsolventen zum Tragen, da in den letzten Jahren ein massiver Wandel in der deutschen Hochschullandschaft stattgefunden hat. Besonders deutlich werden die Veränderungen anhand der neuen Studienabschlüsse Bachelor und Master, die Magister und Diplom zwischenzeitlich weitgehend abgelöst haben. Die Unsicherheit, von der die Rekrutierung von Berufsanfängern ohnehin geprägt ist, wird durch diese Entwicklungen somit noch weiter verstärkt, da sich die neuen Studienabschlüsse von Seiten der Unternehmen derzeitig vielmals nicht sicher einschätzen lassen.

Der Beitrag widmet sich in diesem Sinne der Frage, auf welche Kriterien Entscheidungsträger in Unternehmen bei der Rekrutierung von Hochschulabsolventen achten. Untersucht wird dabei ausschließlich die Gruppe von Absolventen wirtschaftswissenschaftlicher Studiengänge. Auf Grundlage humankapital- und signaltheoretischer Überlegungen wird die Annahme getroffen, dass die individuellen Kompetenzen der Bewerber entscheidend sind. Dazu zählen unter anderen die Abschlussnote und die bereits während des Studiums erworbene Praxiserfahrung, beispielsweise in Form von Praktika oder Nebentätigkeiten. Darüber hinaus wird untersucht, ob zur Verringerung der Unsicherheit die Reputation von Universitäten als Signal in den Auswahlprozess einbezogen wird. Der Ruf einer Organisation kann beispielsweise durch die Presse oder durch Informationen von Personen aus dem persönlichen Netzwerk verbreitet werden. Auch Hochschulrankings, denen seit einiger Zeit in Deutschland eine immer bedeutsamere Rolle zuteilwird, können von Personalentscheidern genutzt werden, um Hinweise auf Inhalt und Qualität einzelner Bildungseinrichtungen zu erhalten. Die auf Grundlage humankapital- und signaltheoretischer Überlegungen aufgestellten Hypothesen werden ergänzt durch theoretische Annahmen zur Bedeutung von Netzwerken und dem Einfluss räumlicher Nähe von Organisationen.

Die empirische Überprüfung der aufgestellten Hypothesen basiert auf den Daten einer bundesweiten Unternehmensbefragung von Fachführungskräften, die in ihrer täglichen Arbeit mit der Einstellung von wirtschaftswissenschaftlichen Hochschulabsolventen betraut sind. Für die Datenerhebung wurde auf ein Switch-Mode-Verfahren zurückgegriffen, das auf einem kombinierten Einsatz von telefonischer Eingangsbefragung und Onlineerhebung beruht. Der Vorteil dieser Herangehensweise liegt darin, dass in einem ersten Schritt ein Unternehmen telefonisch 
kontaktiert und um die Verbindung mit einer geeigneten Zielperson gebeten wird. Dieser wird vom Interviewer eine kurze, themenbezogene Eingangsfrage gestellt. AnschlieBend erfasst der Anrufer die E-Mail-Adresse des Befragten, um die Zugangsdaten für die Teilnahme an der Online-Befragung zuzusenden.

In der Online-Befragung wurde erhoben, welche Kriterien bei der Einstellung von Bewerbern mit wirtschaftswissenschaftlichem Hochschulabschluss wichtig sind. Dabei wurde auf die Methode des faktoriellen Surveys zurückgegriffen. Bei diesem experimentell angelegten Design werden den Teilnehmern fiktive Situationsbeschreibungen zur Bewertung vorgelegt, die Verteilung der einzelne hypothetischen Szenarien, die auch als Vignetten bezeichnet werden, erfolgt randomisiert. Damit lassen sich die kausalen Effekte der in den Situationsbeschreibungen variierten Merkmale auf die Einschätzung der befragten Personen analysieren. In der vorliegenden Erhebung wurde untersucht, welche individuellen Kriterien im Auswahlprozess zählen, und inwiefern der Name der Hochschuleinrichtung, an der ein Bewerber studiert hat, eine Rolle im Auswahlprozess spielt. Hierfür gaben die Befragten für jede Situationsbeschreibung an, wie wahrscheinlich sie den skizzierten Bewerber zu einem Vorstellungsgespräch einladen würden. Um die aktuellen Entwicklungen in der deutschen Hochschullandschaft abzubilden, wurde eine inhaltliche Unterscheidung getroffen: Der Hälfte der Befragten wurden Beschreibungen vorgelegt, in dem die Bewerber über einen Bachelor-Abschluss verfügen. Für die anderen Teilnehmer ging es um die Einschätzung von Hochschulabsolventen mit MasterAbschluss. An der Online-Erhebung beteiligten sich rund 1000 Personen.

Für die multivariate Analyse der Daten wurde aufgrund der Mehrebenenstruktur, die faktoriellen Designs generell zugrunde liegen, auf Random-Intercept-Modelle zurückgegriffen. Die Ergebnisse zeigen, dass die individuelle Qualifikation des Bewerbers entscheidenden Einfluss auf die Erfolgschancen nimmt: Arbeitgeber beziehen die Abschlussnote in ihre Entscheidung ein, allerdings nicht in einem stärkeren Maße als andere individuelle Kriterien. So erhöht insbesondere Praxiserfahrung die Wahrscheinlichkeit zu einem Vorstellungsgespräch eingeladen zu werden. Auch das Vorweisen von Auslandserfahrung und ehrenamtlichem Engagement verbessern die Erfolgschancen. Der Hochschulreputation in Form des Namens der Hochschuleinrichtung, an der ein Bewerber studiert hat, kommt in diesem Prozess nahezu keine Bedeutung zu. Zwar scheinen Entscheidungsträger in Unternehmen bei der Auswahl von Master-Studierenden den Namen der Hochschule, an der ein Bewerber studiert hat, tendenziell eher in ihre Entscheidung einzubeziehen als sie dies bei Bewerbern mit Bachelor-Abschluss tun. Verhältnisse wie in den USA, wo die Reputation von Elite-Universitäten eine große Rolle beim Einstieg in den Arbeitsmarkt spielt, sind in Deutschland auf Grundlage der vorliegenden Ergebnisse allerdings nicht zu finden. Für Unternehmen steht bei der Rekrutierung von Hochschulabsolventen hierzulande weiterhin die individuelle Qualifikation des Bewerbers an erster Stelle.

Danksagung Die Autorin dankt Martin Abraham und Max Kilian sowie den anonymen Gutachtern und den Herausgebern der Zeitschrift für Arbeitsmarktforschung für wertvolle Anmerkungen und Kommentare. Darüber hinaus geht der Dank an die Kurt Glässer-Stiftung für die finanzielle Unterstützung der Studie.

\section{Literatur}

Abraham, M., Grimm, V., Meyer, C., Seebauer, M.: Emergence of Reputation. Forthcoming (2012)

Abraham, M., Meyer, C.: Bedingungen des Erfahrungsaustausches in wirtschaftlichen Transaktionen: Das Beispiel der Reputation von Weiterbildungsanbietern. Z. Soziol. 41(3), 166-181 (2012)

Akerlof, G.A.: The market for „lemons“: quality uncertainty and the market mechanism. Q. J. Econ. 84(3), 488-500 (1970)

Alesi, B., Schomburg, H., Teichler, U.: Humankapitalpotenziale der gestuften Hochschulabschlüsse in Deutschland: Weiteres Studium, Übergang in das Beschäftigungssystem und beruflicher Erfolg von Bachelor-und Master-Absolventen. In: Studien zum deutschen Innovationssystem, 13-2010 (2010)

Altonji, J.G., Pierret, C.R.: Employer learning and statistical discrimination. Q. J. Econ. 116(1), 313-350 (2001)

Arrow, K.J.: Higher education as a filter. J. Public Econ. 2(3), 193-216 (1973)

Auspurg, K., Abraham, M., Hinz, T.: Die Methodik des faktoriellen Surveys in einer Paarbefragung. In: Kriwy, Gross (Hrsg.) Klein aber fein!, S. 179-210. VS, Wiesbaden (2009)

Auspurg, K., Hinz, T., Liebig, S.: Komplexität von Vignetten, Lerneffekte und Plausibilität im Faktoriellen Design. Methoden-DatenAnalysen. 1, 59-96 (2009)

Banscherus, U., Himpele, K., Staack, S.: Akademische Berufsqualifizierung als konzeptionelle Herausforderung an Hochschulen, Politik und Studierendenforschung. WSI-Mitteilungen. 10, 508-514 (2010)

Barney, J.: Firm resources and sustained competitive advantage. J. Manag. 17(1), 99-120 (1991)

Bastedo, M.N., Bowman, N.A.: US news \& world report college rankings: Modeling institutional effects on organizational reputation. Am. J. Educ. 116(2), 163-183 (2010)

Beck, M., Opp, K.-D.: Der faktorielle Survey und die Messung von Normen. Köln. Z. Soziol. Sozialpsychologie. 53(2), 283-306 (2001)

Becker, G.S.: Human capital. Columbia University Press, New York [u. a.] (1964)

Bolton, G.E., Katok, E., Ockenfels, A.: How effective are electronic reputation mechanisms? An experimental investigation. Manag. Sci. 50(11), 1587-1602 (2004)

Bowman, N.A., Bastedo, M.N.: Getting on the front page: Organizational reputation, status signals, and the impact of US news and world report on student decisions. Res. High. Educ. 50(5), 415436 (2009)

Brenzikofer, B.: Reputation von Professoren. Rainer Hampp Verlag, München und Mering (2002)

Burkhardt, A., Schomburg, H., Teichler, U.: Hochschulstudium und Beruf-Ergebnisse von Absolventenstudien. Bonn: Bundesministerium für Bildung und Forschung (BMBF), Referat Öffentlichkeitarbeit (2000) 
Burt, R.S.: Brokerage and closure. University Press, Oxford (2005)

Buskens, V., Weesie, J.: An experiment on the effects of embeddedness in trust situations: Buying a used car. Ration. Soc. 12(2), 227-253 (2000)

DGS: Evaluation ja - CHE-Ranking nein. Methodische Probleme und politische Implikationen des CHE-Hochschulrankings Stellungnahme der DGS. http://www.soziologie.de/uploads/media/Stellungnahme_DGS_zum_CHE-Ranking_Langfassung.pdf (2012). Zugegriffen 02. Juli 2012

Diekmann, A., Wyder, D.: Vertrauen und Reputationseffekte bei Internet-Auktionen. Köln. Z. Soziol. Sozialpsychologie. 54(4), 674$693(2002)$

Dill, D.D., Soo, M.: Academic quality, league tables, and public policy: A cross-national analysis of university ranking systems. High. Educ. 49(4), 495-533 (2005)

Doeringer, P.B., Piore, M.J.: Internal labor markets and manpower analysis. Heath, Lexington (1971)

Ellickson, R.C.: Order without law: How neighbors settle disputes. Harvard University Press, Cambridge (1991)

Falk, S., Reimer, M.: Verschiedene Fächer, verschiedene Übergänge: Der Berufseinstieg und „frühe“ Berufserfolg bayerischer Hochschulabsolventen. Beitr. Hochschulforschung. 29, 34-70 (2007)

Fombrun, C.: Reputation: realizing value from the corporate image. Harvard Business Press, Boston (1996)

Fombrun, C., Riel, C.B.M.: The reputational landscape. Corp. Reput. Rev. 5, 1-16 (1997)

Fombrun, C., Shanley, M.: What's in a name? Reputation building and corporate strategy. Acad. Manag. J. 33, 233-258 (1990)

Franck, E., Opitz, C.: Zur Funktion von Studiengebühren angesichts von Informationsasymmetrien auf Humankapitalmärkten. Z. betriebswirtschaftliche Forsch. 53, 91-106 (2001)

Franck, E., Opitz, C.: Zur Filterleistung von Hochschulsystemen Bildungswege von Topmanagern in den USA, Frankreich und Deutschland. Z. betriebswirtschaftliche Forsch. 56, 72-86 (2004)

Franzen, A., Hecken, A.: Studienmotivation, Erwerbspartizipation und der Einstieg in den Arbeitsmarkt. Köln. Z. Soziol. Sozialpsychologie. 54(4), 733-752 (2002)

Fritsch, M.: Hochschulen, Innovation, Region: Wissenstransfer im räumlichen Kontext. edition sigma, Berlin (2007)

Garibaldi, P.: Personnel economics in imperfect labour markets. University Press, Oxford (2006)

Granovetter, M.: Economic action and social structure: The problem of embeddedness. Am. J. Sociol. 91(3), 481-510 (1985)

Haak, C., Rasner, A.: Search (f) or Work: Der Übergang vom Studium in den Beruf. Köln. Z. Soziol. Sozialpsychologie. 61(2), 235-258 (2009)

Habicht, H.: Universität und Image: Entwicklung und Erprobung eines stakeholderorientierten Erhebungsinstrumentariums. Gabler, Wiesbaden (2009)

Hartmann, M.: Die Exzellenzinitiative - Ein Paradigmenwechsel in der deutschen Hochschulpolitik. Leviathan. 34(4), 447-465 (2006)

Haug, S., Kropp, P.: Soziale Netzwerke und der Berufseinstieg von Akademikern. In: Eine Untersuchung ehemaliger Studierender an der Fakultät für Sozialwissenschaften und Philosophie in Leipzig. Arbeitsbericht des Institutes für Soziologie 32 (2002)

Hazelkorn, E.: The impact of league tables and ranking systems on higher education decision making. High. Educ. Manag. Policy. 19, 87 (2007)

Heine, C., Spangenberg, H., Willich, J.: Informationsbedarf, Informationsangebote und Schwierigkeiten bei der Studien-und Berufswahl. Hochschul Informations System GmbH, Hannover (2007)

Heine, C., Willich, J., Schneider, H.: Informationsverhalten und Hochschulwahl von Studienanfängern in West-und Ostdeutschland. Hochschul-Informations-System GmbH, Hannover (2009)
Höglinger, M., Abraham, M., Arpagaus, J.H., Liechti, L.: Die „Schweizer Betriebsbefragung zur Weiterbildung" - Codebuch. Zürich/Erlangen-Nürnberg: Kaleidos FH/FAU Erlangen-Nürnberg (2010)

Hox, J.J., Kreft, I.G.G., Hermkens, P.L.J.: The analysis of factorial surveys. Sociol. Methods Res. 19, 493 (1991)

Judt, A.: Wie wird der Bachelor von der Wirtschaft aufgenommen? Ergebnisse einer Studie mit den Personalverantwortlichen der Top-1000-Unternehmen in Deutschland. Eine Studie des Fachbereichs Wirtschaftswissenschaften der Universität Frankfurt am Main. http://www.hrk-bologna.de/bologna/de/download/dateien/ Studie_Wiwi_Uni_Frankfurt_2006.pdf (2006). Zugegriffen 11. Sept. 2012

Krawietz, M., Heine, C.: Wahlmotive und Bewertungen des Studienortes bei Studienanfängern im Vergleich der neuen und der alten Länder. HIS: Projektbericht, August (2007)

Landrum, R.E., Turrisi, R., Harless, C.: University image: The benefits of assessment and modeling. J. Mark. Higher Educ. 9(1), 53-68 (1999)

Liebig, S., Mau, S.: Wann ist ein Steuersystem gerecht? Einstellungen $\mathrm{zu}$ allgemeinen Prinzipien der Besteuerung und zur Gerechtigkeit der eigenen Steuerlast. Z Soziol. 34(6), 468-491 (2005)

Lödermann, A.-M., Scharrer, K.: Beschäftigungsfähigkeit von Universitäts absolventen-Anforderungen und Kompetenzen aus Unternehmenssicht. Beitr Hochschulforschung. 32(4), 72-91 (2010)

Lorenz, M., Rohrschneider, U.: Erfolgreiche Personalauswahl. Gabler, Wiesbaden (2009)

Lowry, R.C., Silver, B.D.: A rising tide lifts all boats: Political science department reputation and the reputation of the university. Polit. Sci. Polit. 29(2), 161-167 (1996)

Maas, C.J.M., Hox, J.J.: The influence of violations of assumptions on multilevel parameter estimates and their standard errors. Comput. Stat. Data. Anal. 46, 427-440 (2004)

McDonough, P.M., Lising, A., Walpole, A.M., Perez, L.X.: College rankings: Democratized college knowledge for whom? Res. High. Educ. 39(5), 513-537 (1998)

McGuinness, S.: University quality and labour market outcomes. Appl. Econ. 35(18), 1943-1955 (2003)

McPherson, M., Smith-Lovin, L., Cook, J.M.: Birds of a feather: Homophily in social networks. Annu. Rev. Sociol. 27, 415-444 (2001)

Metropolregion Nürnberg: Die Metropolregion auf einen Blick. http:// www.metropolregionnuernberg.de/region/staedte-landkreise.html (2012). Zugegriffen 21. März 2012

Meyer, C., Schrauth, B., Abraham, M.: Einstellungskriterien für Hochschulabsolventen wirtschaftswissenschaftlicher Studiengänge Ergebnisse einer repräsentativen Umfrage unter Arbeitgebern in Deutschland. LASER, Nürnberg (2013)

Milgrom, P., Roberts, J.: Economics, organization and management. Prentice-Hall, Englewood Cliffs (1992)

Nee, V.: The new instiutionalism in economics and sociology. In: Swedberg, S. (Hrsg.) The handbook of economic sociology. Princeton University Press, Princeton and Oxford (2005)

Nisic, N., Auspurg, K.: Faktorieller Survey und klassische Bevölkerungsumfrage im Vergleich-Validität, Grenzen und Möglichkeiten beider Ansätze. In: Gross, K. (Hrsg.) Klein aber fein! 211-245. VS Verlag für Sozialwissenschaften, Wiesbaden (2009)

Opitz, C.: Hochschulen als Filter für Humankapital: Bildung und Karriere von High Potentials in den USA, Frankreich und Deutschland. Dt. Univ.-Verl, Wiesbaden (2004)

Ott, R.: Darstellung und Beurteilung von Hochschul-Rankings in Deutschland. Beitr. Hochschulforschung. 4, 309-322 (1999)

Raub, W., Weesie, J.: Reputation and efficiency in social interactions: An example of network effects. Am J Sociol. 96, 626-654 (1990)

Rettig, D.: Deutschlands beste Universitäten. Wirtschaftswoche. http:// www.wiwo.de/erfolg/campus-mba/uni-ranking-deutschlands-beste-universitaeten/6476062.html (2012). Zugegriffen 08. Aug. 2012 
Rindova, V.P., Williamson, I.O., Petkova, A.P., Sever, J.M.: Being good or being known: An empirical examination of the dimensions, antecedents, and consequences of organizational reputation. Acad. Manag. J. 48(6), 1033-1049 (2005)

Rooks, G., Raub, W., Selten, R., Tazelaar, F.: How inter-firm co-operation depends on social embeddedness: A vignette study. Acta Sociol. 43(2), 123-137 (2000)

Rossi, P.H., Anderson, A.B.: The factorial survey approach: An introduction. In: Nock, R. (Hrsg.) Measuring social judgments, S. 1567. Sage, Beverly Hills (1982)

Sarcletti, A.: Humankapital und Praktika. Z. Erziehungswissenschaft. 10(4), 549-566 (2007)

Schultz, T.W.: Investment in human capital. Am. Econ. Rev. 51(1), 1-17 (1961)

Schwalbach, J.: Image, Reputation und Unternehmenswert. In: Transnational Communication in Europe. Research and Practice, S. 287-297. Berlin, Vistas (2000)

Shin, C.J., Toutkoushian, R.K.: The past, present, and future of university rankings. In: Shin, J.C., Toutkoushian, R.K., Teichler, U. (Hrsg.) University rankings. Springer, Dordrecht u. a. (2011)

Shrum, W., Wuthnow, R.: Reputational status of organizations in technical systems. Am. J. Sociol. 93, 882-912 (1988)

Sommerfeld, R.D., Krambeck, H.-J., Semmann, D., Milinski, M.: Gossip as an alternative for direct observation in games of indirect reciprocity. Proc. Nat. Acad. Sci. 104(44), 17435 (2007)

Sommerfeld, R.D., Krambeck, H.-J., Milinski, M.: Multiple gossip statements and their effect on reputation and trustworthiness. Proc. Royal Soc. 275(1650), 2529-2536 (2008)

Spence, M.: Job market signaling. Q. J. Econ. 87(3), 355-374 (1973)

Sperling, R.: Berufsfeldorientierte Kompetenzen für Bachelor-Studierende. In: Qualifikationserwartungen von Arbeitgebern an Bachelor-Absolvent (inn) en. Universität Freiburg, BOK-Projekt (2008)
Statistisches Bundesamt: Klassifikation der Wirtschaftszweige. Statistisches Bundesamt, Wiesbaden (2008)

Teichler, U.: Studium und Berufschancen: Was macht den Unterschied aus? Beitr. Hochschulforschung. 29, 10-31 (2007)

Tietzel, M., van der Beek, K., Müller, C.: Was ist eigentlich an den Hochschulen los? Wirtschaftsdienst. 78(3), 148-156 (1998)

Tirole, J.: A theory of collective reputations (with applications to the persistence of corruption and to firm quality). Rev. Econ. Stud. 63(1), 1-22 (1996)

Uzzi, B., Lancaster, R.: Embeddedness and price formation in the corporate law market. Am. Sociol. Rev. 69(3), 319-344 (2004)

Weiss, A.: Human capital vs. signalling explanations of wages. J. Econ. Perspect. 9(4), 133-154 (1995)

Wilson, R.: Reputations in games and markets. In: Roth, A.E. (Hrsg.) Game-theoretic models of bargaining, S. 27-62. Cambridge University Press, Cambridge (1985)

Winston, G.C.: Subsidies, hierarchy and peers: The awkward economics of higher education. J. Econ. Perspect. 13(1), 13-36 (1999)

ZEIT Online. CHE Hochschulranking 2012/13. http://ranking.zeit.de/ che2012/de/ (2012). Zugegriffen 12. Sept. 2012

Christina Neeß, studierte Sozialwissenschaften an der Friedrich-Alexander-Universität Erlangen-Nürnberg und an der Loughborough University, Großbritannien. Von 2007-2013 war sie als wissenschaftliche Mitarbeiterin am Lehrstuhl für Soziologie und Empirische Sozialforschung (Schwerpunkt Arbeitsmarktsoziologie) der Friedrich-Alexander-Universität Erlangen-Nürnberg tätig. Forschungsschwerpunkte: Wirtschafts- und Organisationssoziologie, Arbeitsmarktsoziologie, experimentelle Wirtschafts- und Sozialforschung. 